\title{
The ferns and lycophytes of Reserva Natural Guaricica, Antonina, Paraná, Brazil
}

\author{
Fernando B. Matos, Amabily Bohn, Paulo H. Labiak
}

Universidade Federal do Paraná, Setor de Ciências Biológicas, Departamento de Botânica, Avenida Coronel Francisco Heráclito dos Santos 100, Jardim das Américas, Curitiba, PR, CEP 81.531-980, Brazil.

Corresponding author: Fernando B.Matos, fbtms@yahoo.com.br

\begin{abstract}
We found 204 species of pteridophytes in Reserva Natural Guaricica, a private natural heritage reserve (RPPN) in Antonina, Paraná, Brazil. With approximately 8,600 ha and elevations ranging from sea level to $600 \mathrm{~m}$, RPPN Guaricica has more species of pteridophytes than any other area in Paraná. Ferns are represented by 194 species in 82 genera and 26 families, whereas lycophytes comprise 10 species in four genera and two families. The RPPN is the type locality of two recently described species: Hypolepis acantha Schwartsb. and Oleandra australis Schwartsb. \& J.Prado. It is also the only place of occurrence of Didymoglossum angustifrons Fée, Diplazium riedelianum (Bong. ex Kuhn) C.Chr., Pteris ensiformis Burm.f., P. tripartita Sw., Saccoloma elegans Kaulf., and Steiropteris polypodioides (Raddi) Salino \& T.E.Almeida in Paraná. Pteris ensiformis and Saccoloma brasiliense (C.Presl) Mett. are new state records. Additional species are expected to occur in the area, in view of their known geographical ranges.
\end{abstract}

Keywords

Atlantic rainforest, biodiversity, conservation, floristics, pteridophytes, Rio Cachoeira, SPVS

Academic editor: Denise Pinheiro da Costa | Received 10 September 2019 | Accepted 3 February 2020 | Published 21 February 2020

Citation: Matos FB, Bohn A, Labiak PH (2020) The ferns and lycophytes of Reserva Natural Guaricica, Antonina, Paraná, Brazil. Check List 16 (1): 183-206. https://doi.org/10.15560/16.1.183

\section{Introduction}

Ferns and lycophytes represent distinct evolutionary lineages of vascular plants (Kenrick and Crane 1997; Pryer et al. 2001; PPG I 2016). Despite being distantly related, these two groups are often referred to collectively as "pteridophytes", mainly because they share a unique life cycle with independent gametophyte and sporophyte phases (Haufler et al. 2016). There are about 12,000 species of pteridophytes in the world (PPG I 2016), ca 1,250 of them occurring in Brazil (Prado et al. 2015). Paraná is the fourth richest state of Brazil in terms of diversity of these plants, with estimates ranging from 436 to 491 species (Kaehler et al. 2014; Prado et al. 2015, respectively). Located in the southern hemisphere, between 22 and 27 degrees of latitude, Paraná is biogeographically interesting for being the borderline of distribution of several species, mixing tropical and subtropical elements in its flora (Labiak 2014). There are five main vegetation types within the state (i.e., Araucaria forest, Atlantic forest, Seasonally dry forest, Cerrado, and Campos Gerais), but the great majority of pteridophyte species occur in the Atlantic rainforest (Kaehler et al. 2014). This forest is restricted to the eastern portion of the state, occurring on the coastal plains and along the mountains of Serra do Mar. It can be subdivided into four types 
according to the elevational gradient (i.e., lowland, submontane, montane, and cloud forests), in addition to associated physiognomies such as high-elevation grasslands, mangroves, and restingas (Veloso et al. 1991).

Despite its outstanding biodiversity, the pteridophyte flora of the Atlantic rainforest of Paraná remains poorly studied. Most of the inventories conducted in the state have focused on other phytogeographic domains, such as the Araucaria forest (e.g. Cervi et al. 1987; Dittrich et al. 1999; Borgo and Silva 2003; Kozera et al. 2006; Schwartsburd and Labiak 2007), the Seasonally dry forest (e.g. Cervi and Borgo 2007; Lautert et al. 2015), and the Campos Gerais (e.g. Michelon and Labiak 2013; Michelon et al. 2018). For the Atlantic rainforest we have only the studies by Dittrich et al. (2005) for the Marumbi State Park (1 hectare plot), Salino et al. (2005) for Ilha do Mel, Blum et al. (2011) for Serra da Prata (epiphytes only), and Pereira and Labiak (2018) for Pico Paraná State Park. Surprisingly as it may seem, there are no studies focused on the pteridophytes from the coastal lowlands of Paraná, between the mountains of Serra do Mar and the Atlantic Ocean. Many botanists have visited and collected pteridophytes in this region since the beginning of the 20th century, especially Per Karl Hjalmar Dusén (in 1903-1904, and 1908-1916), Gerdt Guenther Hatschbach (1950-2013), and Paulo Henrique
Labiak Evangelista (1992 to present), but no one has ever published a checklist for this area. Therefore, the purpose of our research is to fill in this gap by presenting a checklist of the ferns and lycophytes from Reserva Natural Guaricica.

\section{Methods}

Study site. The Reserva Natural Guaricica $\left(25^{\circ} 18^{\prime} 53^{\prime \prime} \mathrm{S}\right.$, $\left.048^{\circ} 41^{\prime} 46^{\prime \prime} \mathrm{W}\right)$ is a private natural heritage reserve (RPPN) owned and managed by SPVS (Portuguese acronym for the Society for Wildlife Research and Environmental Education). With approximately 8,600 ha, it is the largest RPPN in the Brazilian state of Paraná, comprising two areas formerly known as Reserva Natural Águas Belas (a.k.a. Gervásio) and Reserva Natural Rio Cachoeira. The whole area is located in the municipality of Antonina, Paraná, Brazil. It is also within the boundaries of the Guaraqueçaba Environmental Protection Area, between the uplands of Serra do Mar (north), the Antonina Bay (south), the Cachoeira river (west), and the Faisqueira river (east) (Fig. 1). Originally, this region was mostly covered by lowland and submontane evergreen rain forests (IBGE 2012), but these ecosystems suffered intense exploitation and many areas were converted to buffalo pastures. Exploitation of mature forests was especially severe in the coastal plains, where

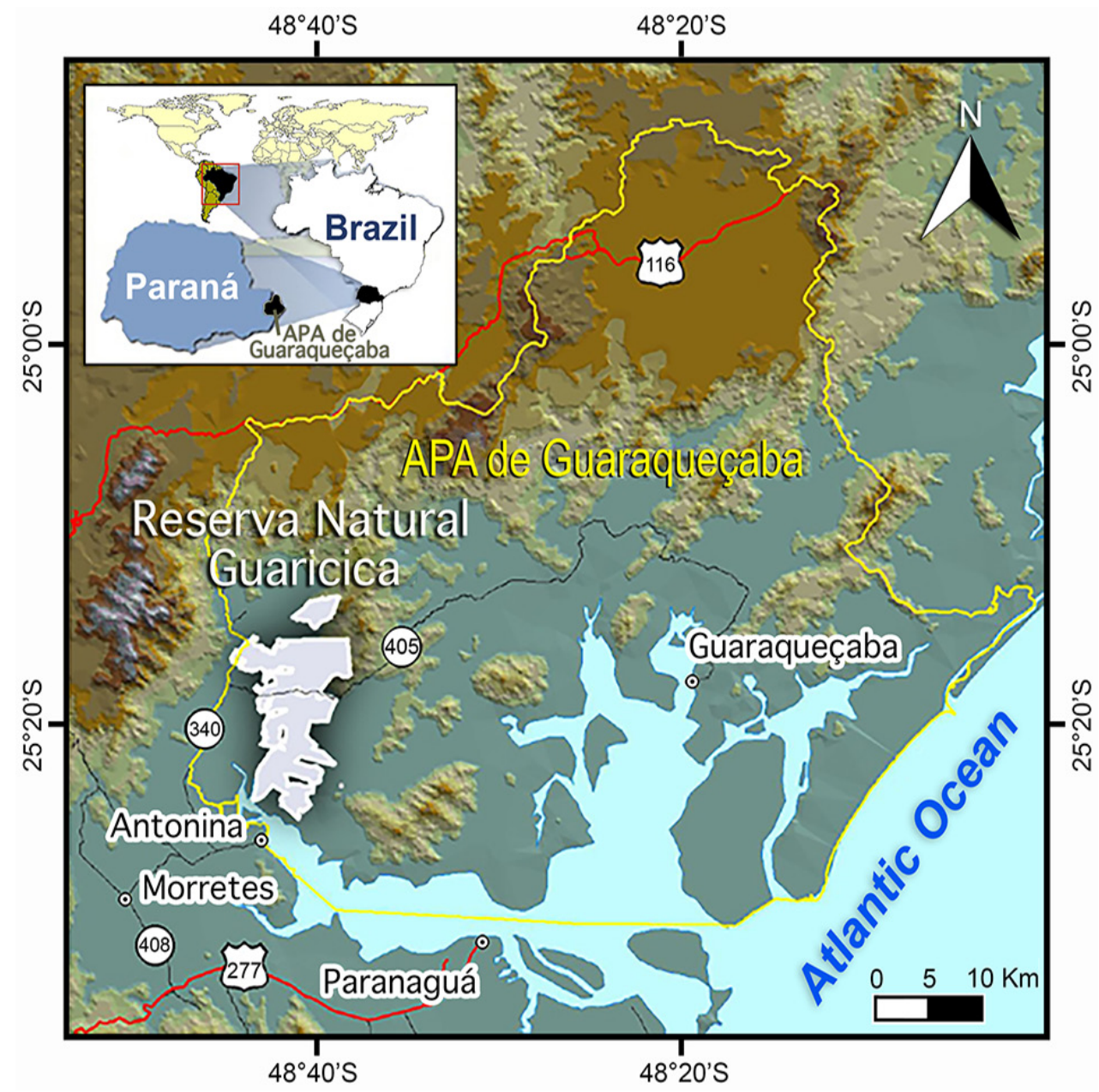

Figure 1. Location map of Reserva Natural Guaricica in the northern coast of Paraná, Brazil. 


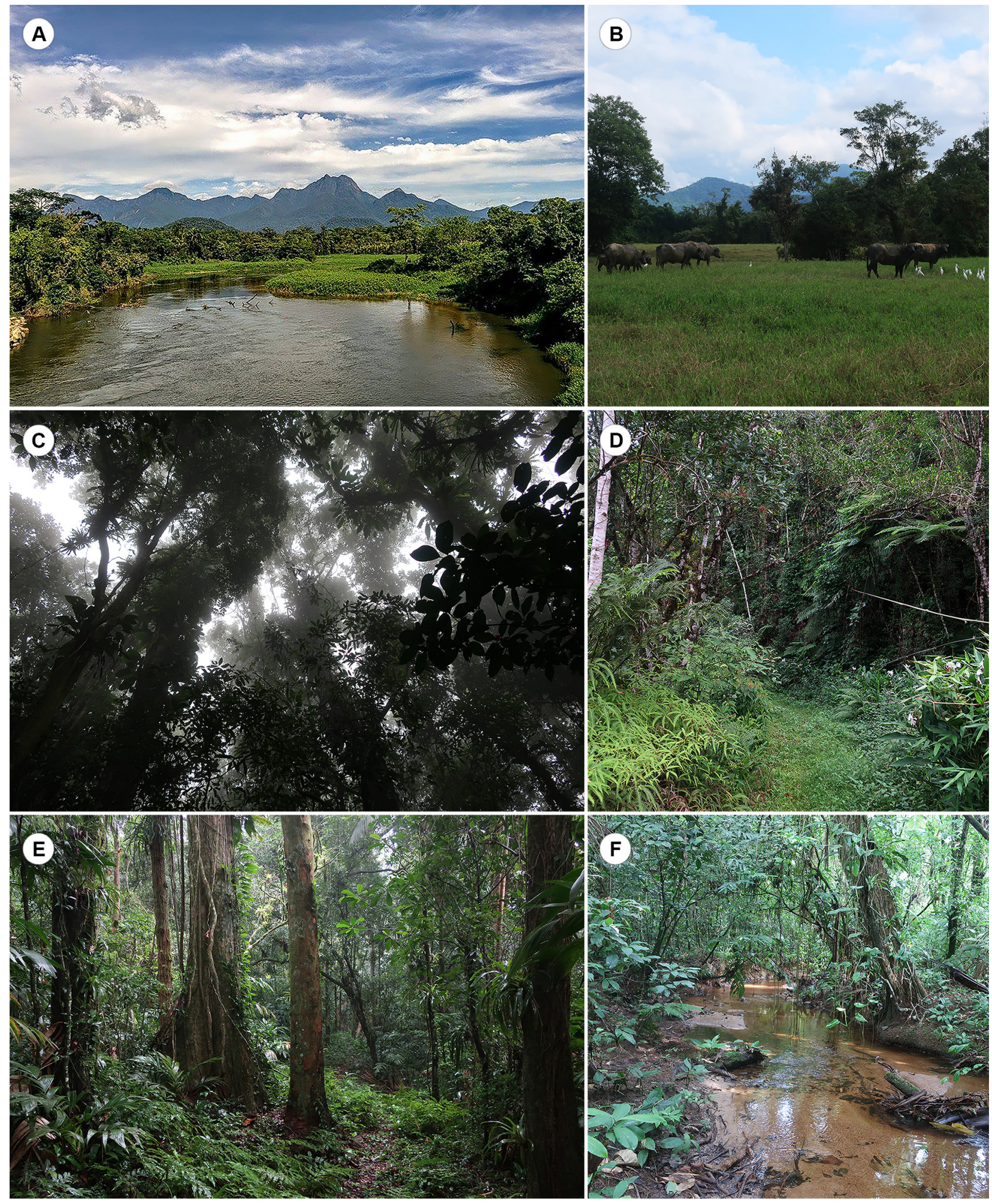

Figure 2. Habitats in Reserva Natural Guaricica. A. Cachoeira River (PR-405), with Pico Paraná State Park on the background. B. Buffalo pasture near the Gervásio area, northern part of the RPPN. C. Canopy of old-grown forest with many epiphytes, trilha da Pantera. D. Disturbed environment along trilha do Gervásio. E. Old-grown submontane forest, trilha da Pantera. F. Stream inside of the forest, trilha do Gervásio. (All photos by FBM, except "A": by Gabriel Inague).

easy access encouraged removal of timber (IPARDES 1995). In 1999, SPVS acquired the whole area and started an ecological restoration program (Ferretti and Britez 2006). The resulting landscape mosaic consists of relatively large patches of old-growth forests, variously aged secondary forests, and pastures. Other vegetation types include riparian forests, mangroves, and restingas (Figs 2, 3). Elevations range from 0 to $600 \mathrm{~m}$ above sea level. The topography varies from coastal plains ( 0 to $20 \mathrm{~m}$, near the Antonina Bay and along Cachoeira and Faisqueira rivers) to isolated hills of about $200 \mathrm{~m}$ and a few higher mountains. The highest mountains are Morro 


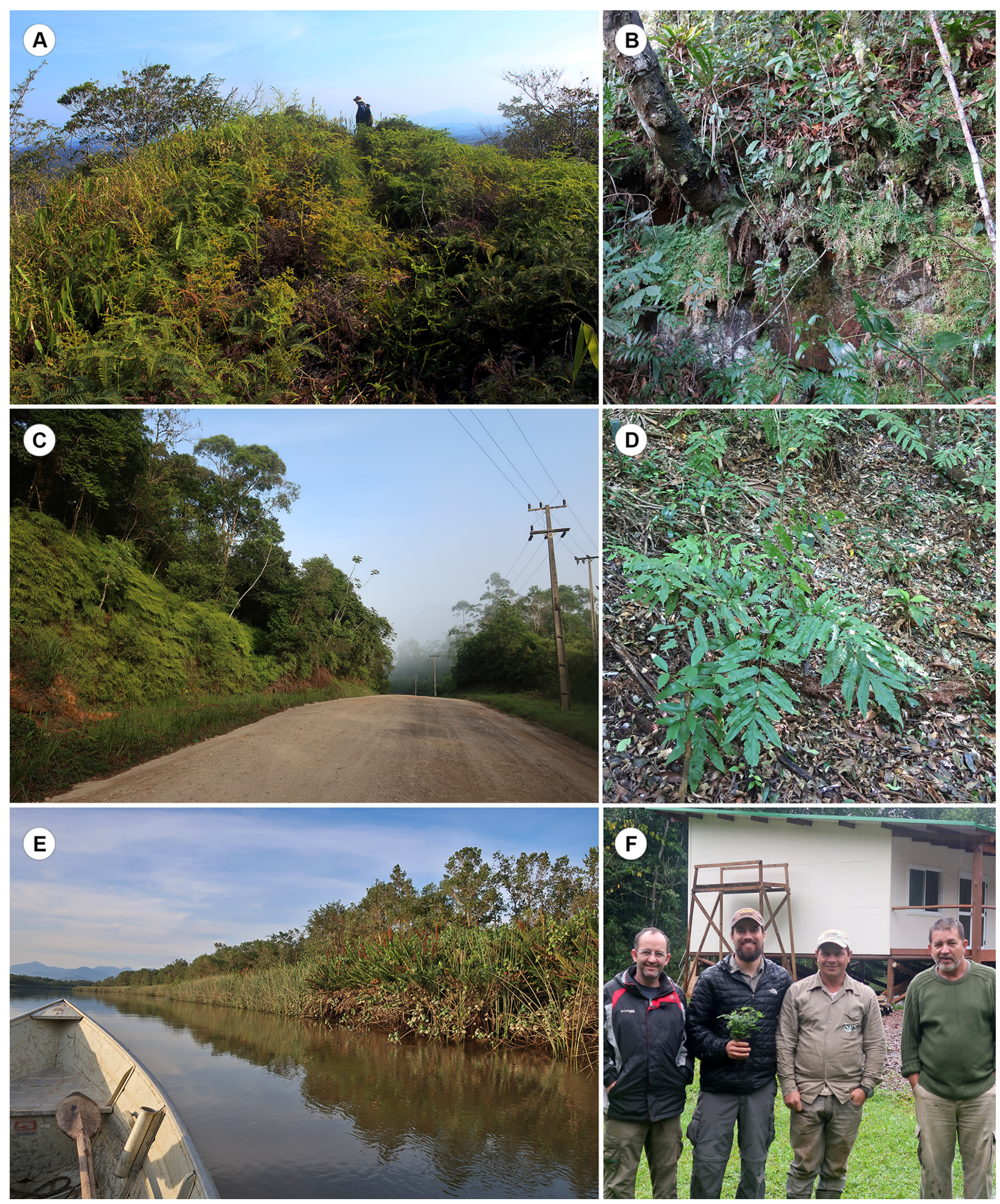

Figure 3. Habitats in Reserva Natural Guaricica. A. Summit of Morro do Mirante, open area with Dicranopteris flexuosa, Palhinhaea cernua, and Pteridium arachnoideum. B. Summit of Morro do Queimado, rocky outrcrop with Oleandra australis, Selaginella flexuosa, and other plants. C. Steep road bank at PR-405 with Gleichenella pectinata. D. Shell midden with Tectaria pilosa, southern part of the RPPN. E. Margin of Cachoeira River with Acrostichum danaeifolium. F. The authors with two of the park rangers that helped tremendously during this study (from left to right: PHL, FBM, João Maria, and João Pontes). (All photos by FBM, except "A": by Maurício Nunes).

do Gervásio (600 m), in the north, and Morro do Queimado (300 m), in the south (i.e., near the Antonina Bay).

The climate is mesothermic humid subtropical (Köppen's Cfa: Kottek et al. 2006), although some authors (e.g. Maack 1981) have classified it as tropical (Köppen's Af). The average annual temperature is between 20.8 and 22
${ }^{\circ} \mathrm{C}$, and the average annual rainfall is $2,545 \mathrm{~mm}$. A relatively marked seasonal variation in rainfall is characteristic of the region, with a concentration of approximately $40 \%$ of annual rainfall in the summer (from January through March) and only 15\% in the driest months (June through August) (Ferretti and Britez 2006). 
Vouchers and data analysis. Most of the collections used for this study were made between the years of 2004 and 2006, when ca 350 specimens were gathered. The identification of these specimens resulted in a preliminary checklist with 196 species for "Reserva Natural Rio Cachoeira" (Matos 2007). Since then, ca 130 samples were collected in seven additional field trips between 2008 and 2019. We have also included ca 150 collections made by other botanists, increasing to 630 the total number of collections used for this study. A complete set of these collections is housed at herbarium UPCB. Duplicates were found mainly at CEPEC, MBM, NY, and RB via SpeciesLink (2019) and Reflora (2019). Herbaria acronyms according to Thiers (2019).

While duplicates of some collections were sent to specialists for identification (see Acknowledgements), the majority of the specimens were identified by us, using the collections at MBM, NY, and UPCB as reference, as well as the main floras and monographs available for the Neotropical region (e.g. Moran 1991; Moran and Riba 1995; Hirai and Prado 2000; Prado and Windisch 2000; Mickel and Smith 2004; Labiak and Prado 2008; Moran et al. 2009; Matos and Mickel 2014; Ø11gaard and Windisch 2019; Viveros et al. 2018).

The checklist is presented in alphabetical order by families, genera, and species. The classification system adopted is that of PPG I (2016). Author abbreviations follow IPNI (2019). Habitat preferences and growth forms were either observed in the field or taken from herbarium labels. Literature was used to access the geographical distribution of each species. To facilitate the future location of taxa in the RPPN, the sampling sites and their respective vegetation types are listed in Table A1. The vegetation characterization of the trails and adjacent areas was performed by analyzing GIS maps that were kindly provided by SPVS, in addition to our own field observations.

Comments and photographs are provided for 27 species. These species represent some of the main orders of ferns and lycophytes, giving an idea of the great morphological diversity found in the area. The following locality applies to all vouchers: "BRAZIL, Paraná, Antonina, Reserva Natural Guaricica". This information was omitted from the citations to avoid repetition.

\section{Results}

We recorded 204 species distributed in 84 genera and 28 families of pteridophytes (Table 1; Figs 4-6). Ferns (Polypodiopsida) comprised 194 species in 80 genera and 26 families, whereas lycophytes (Lycopodiopsida) comprised 10 species in four genera and two

Table 1. Checklist of the ferns and lycophytes from Reserva Natural Guaricica, Antonina, Paraná, Brazil. Sampling sites are those listed in Table A1. Growth forms: $A Q=$ floating aquatic; $A R=$ arborescent; $E P=$ epiphytic; $H E$ = hemiepiphytic; $L I=$ lithophytic; $S C=s c a n d e n t ;$ $\mathrm{TE}=$ terrestrial. Vouchers: FM = Fernando Bittencourt de Matos; MB = Marília Borgo; MG = Marina Caldas Gomes; PL = Paulo Henrique Labiak; PS = Pedro Bond Schwartsburd; RV = Rafael Rosenstock Völtz. A complete set of these vouchers is at UPCB, with duplicates sent to CEPEC, MBM, NY, RB, and SP whenever available. An asterisk (*) indicates that a species is endemic to the Brazilian Atlantic Rainforest; two asterisks $\left(^{* *}\right)$ indicate that the species is endemic to RPPN Guaricica; the word [Exotic] indicates that a species is non-native and invasive.

\begin{tabular}{|c|c|c|c|}
\hline Taxa & Sampling sites & Growth forms & Vouchers \\
\hline \multicolumn{4}{|l|}{ Anemiaceae } \\
\hline Anemia phyllitidis (L.) Sw. & $3,5,22$ & TE & FM 876; MB 2510; MG 47 \\
\hline Anemia tomentosa (Sav.) Sw. var. anthriscifolia (L.) Sw. & 1 & TE & FM 2611 \\
\hline \multicolumn{4}{|l|}{ Aspleniaceae } \\
\hline Asplenium abscissum Willd. & 26 & TE & FM 1197 \\
\hline Asplenium alatum Humb. \& Bonpl. ex Willd. & 24 & TE & FM 1202 \\
\hline Asplenium auriculatum Sw. & 11 & EP & FM 1252 \\
\hline Asplenium auritum Sw. & $4,10,20,36$ & $E P, L I$ & FM 1224, 1272; MB 2111; PL 3022 \\
\hline Asplenium cirrhatum Rich. ex Willd. & $13,17,24$ & EP, LI, TE & FM 566, 1238; MG 4; PL 3940 \\
\hline Asplenium claussenii Hieron. & 11 & TE & FM 1264 \\
\hline Asplenium feei Kunze ex Fée & 11 & EP & FM 1266 \\
\hline Asplenium incurvatum Fée* & 21,24 & EP & FM 1113, 1204 \\
\hline Asplenium kunzeanum Klotzsch ex Rosenst.* & $20,21,35,36$ & EP, TE & FM 135, 476, 2568; MB 2126; MG 3, 41 \\
\hline Asplenium martianum C.Chr.* & 2,36 & EP & FM 201, 546 \\
\hline Asplenium mucronatum C.Presl & $4,21,28,30,35$ & EP & FM 142, 1596, 2571, 2587, 2626; PL 3933; RV 1087 \\
\hline Asplenium pteropus Kaulf. & 3,31 & EP & FM 839; MB 2511; MG 8, 40; PL 3932 \\
\hline Asplenium scandicinum Kaulf. & 26 & EP & FM 148 \\
\hline Asplenium serra Langsd. \& Fisch. & 9,31 & $\mathrm{LI}, \mathrm{TE}$ & FM 1227a, 2640 \\
\hline Asplenium serratum $\mathrm{L}$. & $22,21,26,33$ & EP & FM 153, 194, 1115, 2663 \\
\hline Asplenium uniseriale Raddi & $3,11,28$ & TE & FM 1261, 2597; MB 2500 \\
\hline Hymenasplenium triquetrum (N.Murak. \& R.C.Moran) L.Regalado \& Prada & $11,21,24,31$ & $\mathrm{LI}$ & FM 1127b, 1207, 1241, 1253; MG 46 \\
\hline \multicolumn{4}{|l|}{ Athyriaceae } \\
\hline Deparia petersenii (Kunze) M.Kato [Exotic] & 20 & TE & FM 479 \\
\hline Diplazium ambiguum Raddi & $17,20,21,28,30,36$ & TE & FM 210, 477, 831, 1183, 1601, 2619; MG 14, 32,36 \\
\hline Diplazium cristatum (Desr.) Alston & 24,26 & TE & FM 1198, 1201; PL 3028 \\
\hline Diplazium plantaginifolium (L.) Urb. & $3,19,28,30$ & TE & FM 492, 1599, 2629; MB 2503 \\
\hline
\end{tabular}


Table 1. Continued.

\begin{tabular}{|c|c|c|c|}
\hline Taxa & Sampling sites & Growth forms & Vouchers \\
\hline Diplazium riedelianum (Bong. ex Kuhn) C.Chr. & 28 & TE & FM 1600 \\
\hline \multicolumn{4}{|l|}{ Blechnaceae } \\
\hline Blechnum occidentale L. & 20,22 & TE & FM 866, 1273 \\
\hline Blechnum polypodioides Raddi & $1,12,21,35$ & TE & FM 167, 470, 1594, 2607; MB 2110 \\
\hline Cranfillia mucronata (Fée) V.A.0.Dittrich \& Gasper* & 21 & TE & FM 1116; PL 3936 \\
\hline Lomaridium plumieri (Desv.) C.Presl & 21,26 & HE, TE & FM 158, 2575 \\
\hline Neoblechnum brasiliense (Desv.) Gasper \& V.A.0.Dittrich & $12,15,28$ & TE & FM 472, 1595, 2633 \\
\hline Salpichlaena volubilis (Kaulf.) J.Sm. & $28,35,37$ & SC & FM 206, 1603, 2572 \\
\hline Telmatoblechnum serrulatum (Rich.) Perrie, D.J.Ohlsen \& Brownsey & 22 & TE & FM 183 \\
\hline \multicolumn{4}{|l|}{ Cyatheaceae } \\
\hline Alsophila setosa Kaulf. & 31 & AR & FM 838 \\
\hline Alsophila sternbergii (Sternb.) D.S.Conant & 17,21 & AR & FM 568; RV 1074 \\
\hline Cyathea atrovirens (Langsd. \& Fisch.) Domin & $5,12,17,22$ & AR & FM 181, 199, 469, 550 \\
\hline Cyathea corcovadensis (Raddi) Domin* & 26,30 & AR & FM 155, 2627 \\
\hline Cyathea delgadii Sternb. & 3 & AR & FM 849; RV 1080 \\
\hline Cyathea hirsuta C.Presl* & 21,35 & AR & FM 137, 1268, 2573 \\
\hline Cyathea leucofolis Domin* & 17,21 & AR & FM 136, 571; MG 15, 35 \\
\hline Cyathea phalerata Mart. & 17 & AR & FM 824 \\
\hline \multicolumn{4}{|l|}{ Dennstaedtiaceae } \\
\hline Dennstaedtia cicutaria (Sw.) T.Moore & $2,36,39$ & TE & FM 212, 538, 1132 \\
\hline Dennstaedtia dissecta (Sw.) T.Moore & $17,21,26,39$ & TE & FM 140, 829, 1128, 1194, 2588 \\
\hline Hypolepis acantha Schwartsb.* & 31 & SC & FM 1123; PS 1344 \\
\hline Pteridium arachnoideum (Kaulf.) Maxon & 5,28 & TE & FM 465, 1176 \\
\hline \multicolumn{4}{|l|}{ Didymochlaenaceae } \\
\hline Didymochlaena truncatula (Sw.) J.Sm. & $19,26,33$ & TE & FM 481, 1188, 2668 \\
\hline \multicolumn{4}{|l|}{ Dryopteridaceae } \\
\hline Bolbitis serratifolia (Mert. ex Kaulf.) Schott & 34 & TE & FM 1217 \\
\hline Ctenitis nervata (Fée) R.S.Viveros \& Salino & $9,21,28,31$ & TE & FM 846, 1234, 1242, 2612, 2644 \\
\hline Ctenitis submarginalis (Langsd. \& Fisch.) Ching & $9,28,30$ & TE & FM 2589, 2630, 2646 \\
\hline Elaphoglossum chrysolepis (Fée) Alston* & 21,26 & EP & FM 1110, 1190, 2584; MG 20 \\
\hline Elaphoglossum glabellum J.Sm. & 21,28 & EP & FM 1109, 2592 \\
\hline Elaphoglossum glaziovii (Fée) Brade* & $19,21,36$ & EP & FM 202, 485, 2586; RV 1077 \\
\hline Elaphoglossum lingua Brack. & $17,21,22$ & EP & FM 193, 873, 2582; MB 2027 \\
\hline Elaphoglossum luridum (Fée) Christ & $16,21,22,32,35$ & EP & FM 200, 1235, 2561, 2583, 2585, 2669; MB 1988 \\
\hline Elaphoglossum macahense (Fée) Rosenst. & $14,21,35,33,38$ & EP & FM 197, 1135, 1137, 1223, 2559, 2581, 2599, 2661; PL 3026 \\
\hline Elaphoglossum nigrescens (Hook.) T.Moore ex Diels & 21 & EP & FM 1112; MG 21 \\
\hline Elaphoglossum paulistanum Rosenst.* & 9,11 & EP & FM 1256, 2634 \\
\hline Elaphoglossum rigidum (Aubl.) Urb. & 22 & EP & FM 195 \\
\hline Lastreopsis amplissima (C.Presl) Tindale & 13 & TE & FM 1237 \\
\hline Megalastrum albidum R.C.Moran, J.Prado \& Labiak* & $28,30,31,35$ & TE & FM 841, 1169, 2621; PL 3948, 3984, 3985 \\
\hline Megalastrum connexum (Kaulf.) A.R.Sm. \& R.C.Moran & 21,35 & TE & FM 1104, 1108; PL 3931, 3983 \\
\hline Megalastrum umbrinum (C.Chr.) A.R.Sm. \& R.C.Moran & 26,31 & TE & FM 1126, 1193, 1196; PL 3942 \\
\hline Mickelia scandens (Raddi) R.C.Moran, Labiak \& Sundue* & 17,21 & HE & FM 567; PL 3021 \\
\hline Olfersia corcovadensis Raddi & 31,35 & $\mathrm{LI}, \mathrm{TE}$ & FM 837, 2567; RV 1082 \\
\hline Polybotrya cylindrica Kaulf.* & $14,15,21,22,28,30,31,35$ & HE, TE & FM 141, 840, 1604, 2560, 2578, 2616, 2653; MB 2497; PL 3013, 3952 \\
\hline Rumohra adiantiformis (G.Forst.) Ching & 5 & TE & FM 878 \\
\hline Stigmatopteris bradei Rosenst.* & 13,26 & TE & FM 1240; MG 11, 12 \\
\hline Stigmatopteris caudata (Raddi) C. Chr.* & 19 & TE & FM 484 \\
\hline Stigmatopteris heterocarpa (Fée) Rosenst.* & $20,28,35,36$ & TE & FM 209, 475, 1602, 2569; MG 54; PL 3939 \\
\hline \multicolumn{4}{|l|}{ Gleicheniaceae } \\
\hline Dicranopteris flexuosa (Schrad.) Underw. & 5,28 & TE & FM 461, 462 \\
\hline Gleichenella pectinata (Willd.) Ching & 21 & TE & FM 169 \\
\hline Sticherus bifidus (Willd.) Ching & 5,21 & TE & FM 168, 459 \\
\hline Sticherus lanuginosus (Fée) Nakai & 5 & TE & FM 458 \\
\hline Sticherus nigropaleaceus (J.W.Sturm) J.Prado \& Lellinger & 5 & TE & $\mathrm{FM} \mathrm{457,460}$ \\
\hline \multicolumn{4}{|l|}{ Hemidictyaceae } \\
\hline Hemidictyum marginatum (L.) C.Presl & 26 & TE & FM 1189 \\
\hline \multicolumn{4}{|l|}{ Hymenophyllaceae } \\
\hline Abrodictyum rigidum (Sw.) Ebihara \& Dubuisson & 26 & TE & FM 161; MG 17 \\
\hline Crepidomanes pyxidiferum (L.) Dubuisson \& Ebihara & $17,22,33$ & EP & FM 555, 558, 573, 875, 2660; MG 28 \\
\hline Didymoglossum angustifrons Fée & 22 & EP & FM 874 \\
\hline
\end{tabular}


Table 1. Continued.

\begin{tabular}{|c|c|c|c|}
\hline Taxa & Sampling sites & Growth forms & Vouchers \\
\hline Didymoglossum hymenoides (Hedw.) Copel. & 9,28 & EP & FM 1175, 2643 \\
\hline Didymoglossum krausii (Hook. \& Grev.) C.Presl & $3,10,18,20$ & $E P, L I$ & FM 1228, 1274, 2650; MG 22, 42,43 \\
\hline Hymenophyllum asplenioides (Sw.) Sw. & 28 & EP & FM 1171 \\
\hline Hymenophyllum caudiculatum Mart. & 32 & EP & FM 2672 \\
\hline Hymenophyllum elegans Spreng. & $9,21,28$ & $E P, L I$ & FM 1117, 1121, 2637 \\
\hline Hymenophyllum fragile (Hedw.) C.V.Morton & 28 & EP & FM 1170 \\
\hline Hymenophyllum hirsutum (L.) Sw. & $22,28,33$ & EP & FM 862, 1172, 2664 \\
\hline Hymenophyllum microcarpum Desv. & 26 & EP & FM 1199 \\
\hline Hymenophyllum polyanthos (Sw.) Sw. & 3 & EP & FM 879 \\
\hline Hymenophyllum pulchellum Schltdl. \& Cham. & 28 & EP & FM 1122 \\
\hline Hymenophyllum undulatum (Sw.) Sw. & 21 & EP & FM 1243 \\
\hline Polyphlebium angustatum (Carmich.) Ebihara \& Dubuisson & 19 & EP & FM 489 \\
\hline Polyphlebium diaphanum (Kunth) Ebihara \& Dubuisson & 21,26 & EP, LI & FM 145, 1191, 1244 \\
\hline Polyphlebium hymenophylloides (Bosch) Ebihara \& Dubuisson & 13,39 & EP & FM 1134 \\
\hline Trichomanes cristatum Kaulf. & $18,21,22,28,29$ & TE & FM 186, 1173, 2593, 2652; PL 3961 \\
\hline Trichomanes elegans Rich. & 21,28 & TE & FM 1120, 2576; MG 18; PL 3944 \\
\hline Trichomanes polypodioides $\mathrm{L}$. & $13,17,26,30$ & EP & FM 160, 556 \\
\hline Vandenboschia radicans (Sw.) Copel. & $13,26,30,36$ & HE & FM 149, 157, 2615; MB 2123; PL 3953 \\
\hline \multicolumn{4}{|l|}{ Lindsaeaceae } \\
\hline Lindsaea arcuata Kunze & 11 & TE & FM 1262 \\
\hline Lindsaea divaricata Klotzsch & 17 & TE & FM 548; PL 3937 \\
\hline Lindsaea lancea (L.) Bedd. & $3,17,22,26,35$ & $\mathrm{TE}$ & FM $164,196,561,562,565,853,2565$ \\
\hline Lindsaea portoricensis Desv. & 3,18 & TE & FM 850, 2651 \\
\hline Lindsaea quadrangularis Raddi subsp. terminalis K.U.Kramer & 18,21 & TE & FM 1179, 2648 \\
\hline Lindsaea virescens $\mathrm{Sw} .^{*}$ & 11 & TE & FM 1263 \\
\hline \multicolumn{4}{|l|}{ Lomariopsidaceae } \\
\hline Lomariopsis marginata (Schrad.) Kuhn* & $16,26,35$ & HE & FM 151, 2566; MB 2331; PL 3938 \\
\hline \multicolumn{4}{|l|}{ Lycopodiaceae } \\
\hline Lycopodiella tupiana (B.Øllg. \& P.G.Windisch) B.Øllg. & 5 & TE & FM 463 \\
\hline Palhinhaea cernua (L.) Franco \& Vasc. & $3,5,22,28,35$ & TE & FM 185, 464, 857; PL 3015 \\
\hline Phlegmariurus flexibilis (Fée) B.Øllg.* & $17,21,32$ & EP & FM 834, 2580, 2670 \\
\hline Phlegmariurus heterocarpos (Fée) B.Øllg. & $17,30,35$ & EP & FM 552, 2570, 2624 \\
\hline Phlegmariurus mandiocanus (Raddi) B.Øllg. & $17,21,33,36$ & EP & FM 211, 553, 2577, 2659 \\
\hline \multicolumn{4}{|l|}{ Lygodiaceae } \\
\hline Lygodium volubile Sw. & $5,15,35$ & SC & FM 180, 2655; PL 3025 \\
\hline \multicolumn{4}{|l|}{ Marattiaceae } \\
\hline Danaea geniculata Raddi* & $17,21,26,29,35$ & TE & FM 156, 833, 2564; MG 1; PL 3934, 3972 \\
\hline Danaea moritziana C.Presl* & 21 & TE & FM 1184; MG 7 \\
\hline Danaea nodosa (L.) Sm. & 26 & TE & FM 162; MG 30 \\
\hline Eupodium kaulfussii (J.Sm.) J.Sm.* & 26,30 & TE & FM 163; PL 3034 \\
\hline Marattia cicutifolia Kaulf.* & 11 & TE & FM 1260 \\
\hline \multicolumn{4}{|l|}{ Nephrolepidaceae } \\
\hline Nephrolepis biserrata (Sw.) Schott & 20 & TE & FM 1270 \\
\hline Nephrolepis exaltata (L.) Schott [Exotic] & 39 & TE & FM 1133 \\
\hline Nephrolepis rivularis (Vahl) Mett. ex Krug & $3,19,20,39$ & EP & FM 488, 859, 1129, 1271; PL 3943 \\
\hline \multicolumn{4}{|l|}{ Oleandraceae } \\
\hline Oleandra australis Schwartsb.\&J.Prado** & 9,28 & EP, TE & FM 2635; PL 3941 \\
\hline \multicolumn{4}{|l|}{ Osmundaceae } \\
\hline Osmunda regalis $\mathrm{L}$. & 1 & TE & FM 1592 \\
\hline \multicolumn{4}{|l|}{ Ophioglossaceae } \\
\hline Cheiroglossa palmata (L.) C.Presl & 21,28 & EP & FM 1245, 2595 \\
\hline \multicolumn{4}{|l|}{ Polypodiaceae } \\
\hline Campyloneurum atlanticum R.C.Moran \& Labiak & 17,21 & $E P, L I$ & FM 554, 835; MG 5 \\
\hline Campyloneurum crispum Fée* & 17,22 & $E P, L I$ & FM 198, 574; MG 16 \\
\hline Campyloneurum decurrens (Raddi) C.Presl* & 35 & $\mathrm{LI}$ & FM 1269 \\
\hline Campyloneurum nitidum (Kaulf.) C.Presl & $26,28,29$ & EP & FM 152, 2591; MG 26, 38; PL 3023, 3970 \\
\hline Campyloneurum rigidum J.Sm.* & 22,28 & EP & FM 192, 2590; PL 3032 \\
\hline Ceradenia spixiana (Mart. ex Mett.) L.E.Bishop* & 6 & EP & MB 2247 \\
\hline Cochlidium punctatum (Raddi) L.E.Bishop* & 33 & EP & FM 2658 \\
\hline Cochlidium serrulatum (Sw.) L.E.Bishop & 18,21 & EP & FM 138, 2649; PL 3947 \\
\hline Melpomene melanosticta (Kunze) A.R.Sm. \& R.C.Moran & 28 & EP & FM 2596 \\
\hline
\end{tabular}


Table 1. Continued.

\begin{tabular}{|c|c|c|c|}
\hline Taxa & Sampling sites & Growth forms & Vouchers \\
\hline Microgramma percussa (Cav.) de la Sota & $2,21,28,29$ & EP & FM 545, 1246, 1249, 1598; PL 3963 \\
\hline Microgramma squamulosa (Kaulf.) de la Sota & 9 & EP & FM 2641 \\
\hline Microgramma tecta (Kaulf.) Alston* & $21,30,39$ & EP & FM 1130, 2625; MB 2493; MG 9 \\
\hline Microgramma vacciniifolia (Langsd. \& Fisch.) Copel. & $5,15,16$ & EP & FM 2657; MB 2007, 2137 \\
\hline Pecluma chnoophora (Kunze) Salino \& Costa Assis & $4,22,28$ & EP & FM 187, 2598; MB 1875; RV 1081 \\
\hline Pecluma recurvata (Kaulf.) M.G.Price & $14,17,21$ & EP & FM 551, 1107; MB 1979; MG 25 \\
\hline Pecluma robusta (Fée) M.Kessler \& A.R.Sm. & 3,14 & EP, TE & FM 852, 1136 \\
\hline Pecluma truncorum (Lindm.) M.G.Price & 21,24 & EP & FM 1111, 1180, 1206 \\
\hline Pleopeltis astrolepis (Liebm.) E.Fourn. & $6,14,19,21,29,30,35,38$ & EP & FM 486, 1221, 2558, 2603; MB 2009, 2259; MG 45; PL 3975 \\
\hline Pleopeltis hirsutissima (Raddi) de la Sota & $6,12,15,17,20,28,29$ & EP & FM 468, 480, 827, 1177, 2654; MG 23; PL 3973 \\
\hline Pleopeltis pleopeltifolia (Raddi) Alston & $6,19,21$ & EP & FM 491, 1187, 2606; MG 44; PL 3967 \\
\hline Serpocaulon catharinae (Langsd. \& Fisch.) A.R.Sm. & 12,28 & EP & FM 467, 1118; MG 24 \\
\hline Serpocaulon fraxinifolium (Jacq.) A.R.Sm. & 21 & EP & FM 134, 2579; MB 2495; PL 3033, 3935 \\
\hline Serpocaulon latipes (Langsd. \& Fisch.) A.R.Sm. & 29,38 & TE & FM 1219; PL 3964 \\
\hline Serpocaulon menisciifolium (Langsd. \& Fisch.) A.R.Sm.* & 2,17 & EP & FM 544; MB 2479 \\
\hline Serpocaulon triseriale (Sw.) A.R.Sm. & 29,38 & EP & FM 1222; PL 3974 \\
\hline \multicolumn{4}{|l|}{ Pteridaceae } \\
\hline Acrostichum danaeifolium Langsd. \& Fisch. & $3,7,15,29,34$ & TE & FM 855, 1211, 2656; PL 3981 \\
\hline Adiantopsis chlorophylla (Sw.) Fée & 10 & TE & FM 1226 \\
\hline Adiantum abscissum Schrad. & $9,19,30$ & TE & FM 487, 2623, 2645 \\
\hline Adiantum humile Kunze & 2,22 & TE & FM 547, 877, 2617 \\
\hline Adiantum obliquum Willd. & 25 & TE & FM 1229 \\
\hline Adiantum pentadactylon Langsd. \& Fisch.* & 29,34 & TE & FM 1214; PL 3980 \\
\hline Adiantum raddianum C.Presl & 5 & TE & FM 2613 \\
\hline Adiantum terminatum Kunze ex Miq. & $17,30,31,37$ & TE & FM 208, 570, 847 \\
\hline Adiantum tetraphyllum Willd. & 30 & TE & MG 48 \\
\hline Doryopteris nobilis (T. Moore) C. Chr.* & 10 & TE & FM 1225 \\
\hline Doryopteris pentagona Pic.Serm. & $25,33,34$ & TE & FM 1215, 1230, 2666; MG 50; PL 3036 \\
\hline Doryopteris sagittifolia (Raddi) J.Sm. & $13,17,30,34$ & $\mathrm{LI}, \mathrm{TE}$ & FM 569, 1216, 1239; MB 2151 \\
\hline Hecistopteris pumila (Spreng.) J.Sm. & $3,17,21,33$ & EP & FM 139, 836, 882, 2662; PL 3946 \\
\hline Hemionitis tomentosa (Lam.) Raddi & 34 & TE & FM 1210; PL 3029 \\
\hline Pityrogramma calomelanos (L.) Link & 5 & TE & FM 473 \\
\hline Polytaenium cajenense (Desv.) Benedict & 17,30 & EP & FM 564; MB 2140; MG 6, 39 \\
\hline Polytaenium citrifolium (L.) Schuettp. & $17,26,33,37$ & EP & FM 150, 207, 563, 828, 2667 \\
\hline Pteris altissima Poir. & $24,26,31$ & TE & FM 842, 1195, 1208; PL 3018, 3031, 3945 \\
\hline Pteris decurrens C. Presl & $19,21,30$ & TE & FM 482, 1106, 1181, 2620; PL 3016 \\
\hline Pteris deflexa Link & 24 & TE & FM 1200 \\
\hline Pteris ensiformis Burm.f. [Exotic] & 30 & TE & MG 51 \\
\hline Pteris splendens Kaulf. & $11,24,30$ & TE & FM 1203, 1265, 2618 \\
\hline Pteris tripartita Sw. [Exotic] & 20 & TE & FM 474 \\
\hline Pteris vittata L. [Exotic] & 1,6 & TE & FM 2605, 2614 \\
\hline Radiovittaria stipitata (Kunze) E.H.Crane & $4,6,22,26,29,32$ & EP & FM 147, 188; MB 1874, 2246, 2671; PL 3966 \\
\hline Vittaria graminifolia Kaulf. & 17 & EP & FM 559 \\
\hline Vittaria lineata (L.) Sm. & $11,26,30$ & EP & FM 146, 1258 \\
\hline \multicolumn{4}{|l|}{ Saccolomataceae } \\
\hline Saccoloma brasiliense (C.Presl) Mett.* & 21 & TE & FM 1178; MG 19; PL 3954 \\
\hline Saccoloma elegans Kaulf. & 39 & TE & FM 1131 \\
\hline \multicolumn{4}{|l|}{ Salviniaceae } \\
\hline Azolla filiculoides Lam. & 22 & $\mathrm{AQ}$ & FM 863 \\
\hline Salvinia auriculata Aubl. & $7,21,22,29$ & $\mathrm{AQ}$ & FM 871, 2574; PL 3982 \\
\hline \multicolumn{4}{|l|}{ Schizaeaceae } \\
\hline Actinostachys pennula (Sw.) Hook. & 3 & TE & FM 880 \\
\hline Schizaea elegans (Vahl) Sw. & 9,28 & TE & FM 1119, 2594, 2636; PL 3950 \\
\hline \multicolumn{4}{|l|}{ Selaginellaceae } \\
\hline Selaginella flexuosa Spring & 9,21 & TE & FM 166, 2638 \\
\hline Selaginella macrostachya (Spring) Spring* & 11 & TE & FM 1259 \\
\hline Selaginella muscosa Spring & 3 & TE & FM 883,885 \\
\hline Selaginella sulcata (Desv. ex Poir.) Spring ex Mart. & 22 & TE & FM 865 \\
\hline Selaginella valida Alston* & 17 & TE & FM 557,826 \\
\hline \multicolumn{4}{|l|}{ Tectariaceae } \\
\hline Tectaria incisa Cav. & $16,21,29$ & TE & FM 143, MB 2311, PL 3024, 3962 \\
\hline
\end{tabular}


Table 1. Continued.

\begin{tabular}{|c|c|c|c|}
\hline Taxa & Sampling sites & Growth forms & Vouchers \\
\hline Tectaria pilosa (Fée) R.C.Moran & 19,33 & TE & FM 490, 2665 \\
\hline Tectaria vivipara Jermy \& T.G.Walker & 21 & TE & FM 1182, PL 3949 \\
\hline \multicolumn{4}{|l|}{ Thelypteridaceae } \\
\hline Amauropelta araucariensis (Ponce) Salino \& T.E.Almeida* & 3,29 & TE & FM 854; PL 3965, 3976 \\
\hline Amauropelta opposita (Vahl) Pic.Serm. & 12,29 & TE & FM 205, PL 3014, 3969 \\
\hline Amauropelta ptarmica (Kunze ex Mett.) Pic.Serm.* & 26 & TE & FM 1192 \\
\hline Amauropelta raddii (Rosenst.) Salino \& T.E.Almeida* & $21,26,28,34$ & TE & FM 154, 1185, 1213, 1236, 1605 \\
\hline Christella conspersa (Schrad.) Á.Löve \& D.Löve & 2 & TE & FM 541 \\
\hline Christella dentata (Forssk.) Brownsey \& Jermy [Exotic] & 35 & TE & FM 2674 \\
\hline Christella hispidula (Decne.) Holttum [Exotic] & $2,3,5,6,22,31,35$ & TE & FM 466, 539, 543, 858, 867, 1124, 2604, 2673 \\
\hline Christella patens (Sw.) Pic.Serm. & 34,38 & TE & FM 1212, 1218 \\
\hline Cyclosorus interruptus (Willd.) H.Ito & 2 & TE & FM 542 \\
\hline Goniopteris burkartii C.Chr. ex Abbiatti & $2,22,29$ & TE & FM 870, 1250, PL 3960 \\
\hline Goniopteris lugubris (Mett.) Brade & 3,21 & TE & FM 884, 1105; MG 10; PL 3019 \\
\hline Goniopteris paranaensis (Salino) Salino \& T.E.Almeida* & $9,17,22,28,31$ & TE & FM 575, 823, 844, 868, 1606, 2647; MG 31 \\
\hline Goniopteris riograndensis (Lindm.) Ching & 3 & TE & FM 860 \\
\hline Goniopteris vivipara (Raddi) Brade* & $19,29,30$ & TE & FM 483, 2628; PL 3971, 3977 \\
\hline Macrothelypteris torresiana (Gaudich.) Ching [Exotic] & 3 & TE & FM 848 \\
\hline Meniscium maxonianum (A.R.Sm.) R.S.Fernandes \& Salino & 21 & TE & FM 1593 \\
\hline Meniscium longifolium Desv. & $14,17,22,38$ & TE & FM 189, 825, 1138, 1220 \\
\hline Meniscium serratum Cav. & 2,20 & TE & FM 478, 540 \\
\hline Steiropteris decussata (L.) A.R.Sm. & $12,21,22$ & TE & FM 184, 471, 1233, 1609 \\
\hline Steiropteris polypodioides (Raddi) Salino \& T.E.Almeida & $17,30,35$ & TE & FM 832, 2563, 2622; MG 49 \\
\hline
\end{tabular}

families (Fig. 7A). Among the ferns, the most representative family was Pteridaceae with 27 species, followed by Polypodiaceae (25 spp.), Dryopteridaceae (23 spp.), Hymenophyllaceae (21 spp.), Thelypteridaceae (20 spp.), and Aspleniaceae (17 spp; Fig. 7B). These six families together correspond to ca $65 \%$ of the pteridophyte species found in the reserve. The lycophytes were represented by Lycopodiaceae and Selaginellaceae (five species each). The most diverse genera were Asplenium L. (16 spp.), Elaphoglossum Schott ex J.Sm. and Hymenophyllum Sm. (9 spp. each), Adiantum L. and Pteris L. (7 spp. each), Cyathea Sm. and Lindsaea Dryand. ex Sm. (6 spp. each), and Campyloneurum C.Presl, Goniopteris C.Presl, Selaginella P.Beauv., and Serpocaulon A.R.Sm. (5 spp. each; Fig. 7C).

The most common growth form was terrestrial (109 species, ca 53.4\%), followed by epiphytic (63 spp., $30.9 \%$ ), arborescent (tree ferns) (8 spp., 3.9\%), hemiepiphytic (4 spp., 1.9\%), scandent (3 spp., 1.5\%), litophytic (2 spp., 1\%), and floating aquatic (2 spp., 1\%). Other 13 species $(6.3 \%)$ were observed on more than one substrate (Fig. 7D). Polybotrya cylindrica Kaulf. (Fig. 6D) was observed as hemiepiphytic and terrestrial, but the fertile individuals were always hemiepiphytic.

Regarding the geographic distribution, RPPN Guaricica has 43 species (21\%) that are endemic to the Brazilian Atlantic rainforest (Table 1, asterisks). The following eight species are non-native and invasive: Christella dentata (Forssk.) Brownsey \& Jermy, C. hispidula (Decne.) Holttum (Fig. 5I), Deparia petersenii (Kunze) M.Kato, Macrothelypteris torresiana (Gaudich.) Ching, Nephrolepis exaltata (L.) Schott (Fig. 6E), Pteris ensiformis Burm.f., P. tripartita Sw., and P. vittata L. The
RPPN Guaricica is the type locality of Hypolepis acantha Schwartsb. and Oleandra australis Schwartsb. \& J.Prado (which is known exclusively from the RRPN; Figs 3B, 6G) (Schwartsburd 2012; Schwartsburd et al. 2016, respectively). It is also the only place of occurrence of Didymoglossum angustifrons Fée, Diplazium riedelianum (Bong. ex Kuhn) C.Chr., Pteris ensiformis Burm.f., P. tripartita Sw., Saccoloma elegans Kaulf. (Fig. 4I), and Steiropteris polypodioides (Raddi) Salino $\&$ T.E.Almeida in the state of Paraná. Two species, Pteris ensiformis and Saccoloma brasiliense (C.Presl) Mett., are here recorded for the first time in Paraná (based on Kaehler et al. 2014). Comments and photographs are provided for the 27 species below.

Class Lycopodiopsida Bartl.

Order Lycopodiales DC. ex Bercht. \& J.Presl, Lycopodiaceae P.Beauv.

Palhinhaea cernua (L.) Franco \& Vasc.

Figures 3A, 4A

Examined material. BRAZIL • 2 specimens; Trilha da Guaricica; $25^{\circ} 19^{\prime} 13^{\prime \prime} \mathrm{S}, 048^{\circ} 42^{\prime} 04^{\prime \prime} \mathrm{W}$; alt. $30 \mathrm{~m}$; 15 Jan. 2005; F.B. Matos 185 leg.; MBM333021, UPCB10699 •

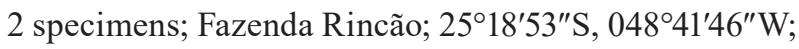
alt. 20 m; 1 Mar. 2005; F.B. Matos \& U. Ferreira 464 leg.; MBM333022, UPCB10700 - 3 specimens; Faisqueira; $25^{\circ} 22^{\prime} 14^{\prime \prime} \mathrm{S}, 048^{\circ} 39^{\prime} 56^{\prime \prime} \mathrm{W}$; alt. 15 m; 7 Oct. 2005; F.B. Matos \& P.B. Schwartsburd 857 leg.; CEPEC118605, RB726314, UPCB10781 • 1 specimen; Fazenda da SPVS; $25^{\circ} 18^{\prime} \mathrm{S}, 048^{\circ} 41^{\prime} \mathrm{W}$; alt. 40 m; 20 Oct. 2003; P.H. Labiak \& R. Goldenberg 3015 leg.; UPCB10786.

Identification. Characterized by anisotomously branched 


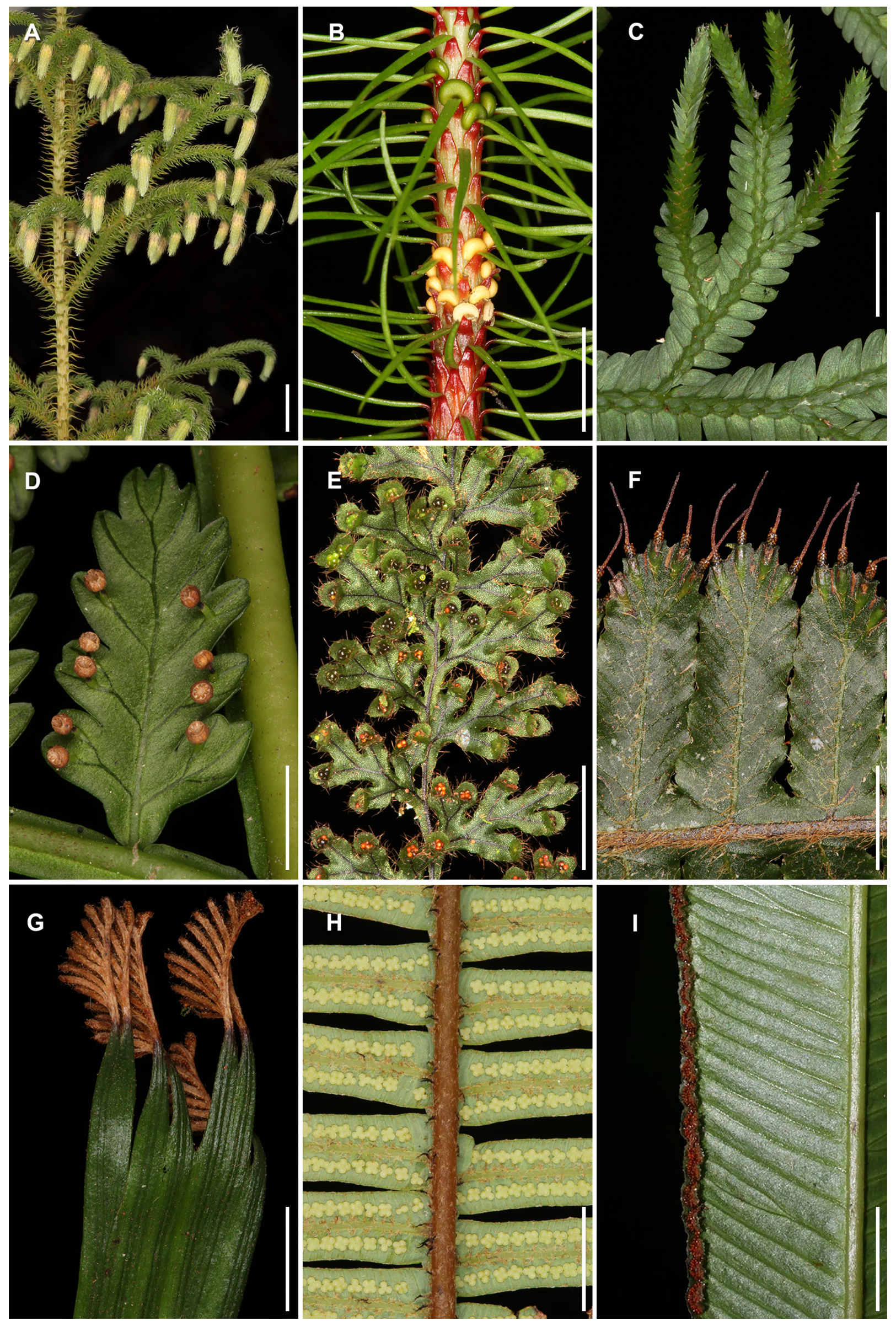

Figure 4. Some lycophytes and ferns from RPPN Guaricica. A. Palhinhaea cernua. B. Phlegmariurus mandiocanus. C. Selaginella flexuosa. D. Eupodium kaulfussii. E. Hymenophyllum elegans. F. Trichomanes cristatum. G. Schizaea elegans. H. Sticherus nigropaleaceus. I. Saccoloma elegans. (All photos by FBM, except H, by Nathan Smith). Scale bars: $0.5 \mathrm{~cm}(\mathbf{B}, \mathbf{D}, \mathbf{E}, \mathbf{F}, \mathbf{H}, \mathbf{I}) ; 1 \mathrm{~cm}(\mathbf{A}, \mathbf{C}, \mathbf{G})$. 
stems, the branches differentiated into horizontal stolon-like stems and vertical tree-like stems. The tree-like stems are usually erect and crowded with small (ca 3 $\mathrm{mm}$ ) linear leaves, with spreading lateral branches ending in sessile, pendent, cylindrical strobili. Sporangia globose, anisovalvate, solitary in the axil of sporophylls. Plants homosporous. It is the only species of the genus in the RPPN.

Distribution and habitat. Pantropical. Terrestrial on roadbanks and other disturbed places.

\section{Phlegmariurus mandiocanus (Raddi) B.Øllg. Figure 4B}

Examined material. BRAZIL - 2 specimens; Trilha

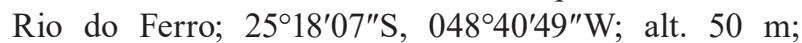
16 Jan. 2005; F.B. Matos 211 leg.; MBM333017, UPCB10953 • 1 specimen; Trilha do Corvo; 2519'39"S, $048^{\circ} 40^{\prime} 26^{\prime \prime} \mathrm{W}$; alt. 100 m; 1 May 2005; F.B. Matos et al. 553 leg.; UPCB10955 • 1 specimen; Trilha do Gervásio; 2514'39"S, 048 39'59"W; alt. 100 m; 27 Aug. 2017; F.B. Matos et al. 2577 leg.; UPCB44063 • 1 specimen; Trilha Porto do Rio Seco; 2524'19"S, 048 40'54"W; alt. 10 m; 25 Jun. 2019; F.B. Matos \& V. Veiga 2659 leg.; UPCB43775.

Identification. Characterized by isotomously branched stems, up to seven times dichotomous. The stems are erect to pendulous, with alternating whorls of spreading, linear leaves ca 10-25 mm long. Sporangia reniform, isovalvate, solitary in the axil of sporophylls. Plants homosporous. Phlegmariurus mandiocanus differs from the other two species of the genus in the area by having the stems almost completely covered by brightly red, decurrent leaf bases.

Distribution and habitat. Paraguay, N Argentina and Brazil. Epiphytic in forests.

Order Selaginellales Prantl, Selaginellaceae Willk.

\section{Selaginella flexuosa Spring}

Figures 3B, 4C

Examined material. BRAZIL -3 specimens; Trilha do Gervásio; 2514'39"S, 048 39'59"W; alt. 50 m; 21 Dec. 2004; F.B. Matos \& P.H. Labiak 166 leg.; CEPEC122125, MBM333141, RB691218 • 1 specimen; Morro do Queimado; $25^{\circ} 23^{\prime} 10^{\prime \prime} \mathrm{S}, 048^{\circ} 42^{\prime} 19^{\prime \prime} \mathrm{W}$; alt. $300 \mathrm{~m}$; 12 Jun. 2019; F.B. Matos et al. 2638 leg.; UPCB44416.

Identification. Characterized by dichotomous, usually prostrate, non-articulate stems with 4 ranks of leaves: two lateral rows of larger spreading leaves and two rows of appressed and ascending median leaves on upper side of stem. Sporangia globose, opening by a slit across the top, arranged in quadrangular strobili located terminally on axes. Plants heterosporous, the megaspores four per sporangium, white, the microspores numerous per sporangium, orange. Sellaginella flexuosa differs from other species of the genus in the area by oblong to elliptic lateral leaves, aristate median leaves, and a somewhat rugose texture of leaves adaxially.

Distribution and habitat. Neotropical. Terrestrial or rarely epiphytic in forests.

Class Polypodiopsida Cronquist, Takht. \& W.Zimm.

Order Marattiales Link, Marattiaceae Kaulf.

\section{Eupodium kaulfussii (J.Sm.) J.Sm.}

Figure 4D

Examined material. BRAZIL - 2 specimens; Trilha do Meio; 25 $14^{\prime} 38^{\prime \prime} \mathrm{S}, 048^{\circ} 39^{\prime} 13^{\prime \prime} \mathrm{W}$; alt. $100 \mathrm{~m}$; 21 Dec. 2004; F.B. Matos \& P.H. Labiak 163 leg.; MBM333104; UPCB6231 - 1 specimen; Fazenda da SPVS; $25^{\circ} 18^{\prime} \mathrm{S}$, $048^{\circ} 41^{\prime} \mathrm{W}$; alt. $50 \mathrm{~m}$; 20 Oct. 2003; P.H. Labiak \& R. Goldenberg 3034 leg.; UPCB6225.

Identification. Characterized by globose stems, bearing only one leaf (rarely two) per plant, ear-like stipules at the base of each leaf, 3-pinnate-pinnatifid blades, and stalked synangia. The most similar species in the area is Marattia cicutifolia Kaulf., which differs by having several leaves per plant, 2-pinnate blades, and sessile synangia.

Distribution and habitat. Endemic to Brazil, from Bahia to Rio Grande do Sul. Terrestrial in forests.

Order Hymenophyllales A.B.Frank, Hymenophyllaceae Mart.

\section{Hymenophyllum elegans Spreng.}

Figure 4E

Examined material. BRAZIL 1 specimen; Trilha do Gervásio; 25¹4'39"S, 048³9'59"W; alt. 300 m; 25 Mar. 2006; F.B. Matos et al. 1117 leg.; UPCB5356 • 2 spec-

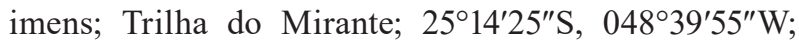
alt. 250 m; 25 Mar. 2006; F.B. Matos et al. 1121 leg.; CEPEC118539, RB648457 • 1 specimen; Morro do Queimado; $25^{\circ} 23^{\prime} 10^{\prime \prime} \mathrm{S}, 048^{\circ} 42^{\prime} 19^{\prime \prime} \mathrm{W}$; alt. $300 \mathrm{~m}$; 12 Jun. 2019; F.B. Matos et al. 2637 leg.; UPCB43865.

Identification. Characterized by filiform stems ca 0.2 $\mathrm{mm}$ in diameter, pendent leaves up to $8 \mathrm{~cm}$ long, nonalate petioles, and linear to lanceolate blades that are 1-pinnate-pinnatifid at base, pinnae mostly adnate, and rachis not alate at base. Also characteristic is the presence of simple, forked or stellate hairs on the leaf. Sori marginal, round, indusia consisting of two lobes. Other hairy species of Hymenophyllum, such as $H$. fragile, $H$. hirsutum and $H$. pulchellum, differ by petiolulate pinnae (at least the lower pairs) or rachises alate to base.

Distribution and habitat. Neotropical. Epiphytic or epipetric in forests.

\section{Trichomanes cristatum Kaulf.}

Figure 4F

Examined material. BRAZIL 1 specimen; Trilha da Guaricica; $25^{\circ} 19^{\prime} 13^{\prime \prime} \mathrm{S}, 048^{\circ} 42^{\prime} 04^{\prime \prime} \mathrm{W}$; alt. 30 m; 15 Jan. 2005; F.B. Matos 186 leg.; UPCB5701 • 1 specimen; Trilha do Mirante; $25^{\circ} 14^{\prime} 25^{\prime \prime} \mathrm{S}, 048^{\circ} 39^{\prime} 55^{\prime \prime} \mathrm{W}$; alt. 360 
m; 27 May 2006; F.B. Matos \& M.C. Gomes 1173 leg.; UPCB5694 - 1 specimen; Trilha do Mirante; 2514'25"S, $048^{\circ} 39^{\prime} 55^{\prime \prime} \mathrm{W}$; alt. 350 m; 27 Aug. 2017; F.B. Matos et al. 2593 leg.; UPCB44197 • 1 specimen; Trilha Esborracha Faisqueira; $25^{\circ} 23^{\prime} 37^{\prime \prime} \mathrm{S}, 048^{\circ} 40^{\prime} 52^{\prime \prime} \mathrm{W}$; alt. $15 \mathrm{~m}$; 12 Jun. 2019; F.B. Matos et al. 2652 leg.; UPCB44480 • 2 specimens; Trilha do Neno; $25^{\circ} 22^{\prime} 36^{\prime \prime} \mathrm{S}, 048^{\circ} 40^{\prime} 10^{\prime \prime} \mathrm{W}$; alt. 10 m; 28 Jun. 2007; P.H. Labiak et al. 3961 leg.; NY886647, UPCB5707.

Identification. Characterized by short-creeping stems, linear to narrowly lanceolate leaves, deeply pinnatifid blades, and long hairs (up to $6 \mathrm{~mm}$ ) adpressed to the rachises and petioles. Sori marginal, in tubular indusia at the apex of primary segments, receptacles usually exerted beyond indusia. This species is so locally common and distinct that it has received its own vernacular name: "rooster-tail fern".

Distribution and habitat. Widely distributed in South America, but apparently absent in Amazon lowlands. Terrestrial in forests.

Order Gleicheniales Schimp., Gleicheniaceae C.Presl

\section{Sticherus nigropaleaceus (J.W.Sturm) J.Prado \& Lellinger}

Figure $4 \mathrm{H}$

Examined material. BRAZIL - 1 specimen; Fazenda Rincão; $25^{\circ} 18^{\prime} 53^{\prime \prime} \mathrm{S}, 048^{\circ} 41^{\prime} 46^{\prime \prime} \mathrm{W}$; alt. $30 \mathrm{~m}$; 1 Mar. 2005; F.B. Matos \& U. Ferreira 457 leg.; UPCB5104 • 1 specimen; same collection data as for preceding; F.B. Matos \& U. Ferreira 460 leg.; UPCB5111.

Identification. Characterized by long-creeping, scaly stems, 1-3 times pseudodichotomously forked leaves, pinnae opposite with a scaly resting bud between the forks, and penultimate segments pectinate. Pseudostipules also present between forks. Sori round, not indusiate. Sticherus nigropaleaceus differs from other species of the genus by having blackish scales on buds and rachises, as well as trifid pseudostipules.

Distribution and habitat. Endemic to Brazil, from Bahia to Rio Grande do Sul. Terrestrial along roads, trails, and forest edges.

Order Schizaeales Schimp., Schizaeaceae Kaulf.

\section{Schizaea elegans (Vahl) Sw.}

Figure $4 \mathrm{G}$

Examined material. BRAZIL • 3 specimens; Trilha do Mirante; $25^{\circ} 14^{\prime} 25^{\prime \prime} \mathrm{S}, 048^{\circ} 39^{\prime} 55^{\prime \prime} \mathrm{W}$; alt. 300 m; 25 Mar. 2006; F.B. Matos et al. 1119 leg.; MBM333148, RB690975, UPCB9981 - 1 specimen; Trilha do Mirante; 2514'25"S, $048^{\circ} 39^{\prime} 55^{\prime \prime}$ W; alt. 350 m; 27 Aug. 2017; F.B. Matos et al. 2594 leg.; UPCB44385 • 1 specimen; Morro do Queimado; $25^{\circ} 23^{\prime} 10^{\prime \prime} \mathrm{S}, 048^{\circ} 42^{\prime} 19^{\prime \prime} \mathrm{W}$; alt. $300 \mathrm{~m}$; 12 Jun. 2019; F.B. Matos et al. 2636 leg.; UPCB44486 - 2 specimens; $25^{\circ} 14^{\prime} 28^{\prime \prime} \mathrm{S}, 048^{\circ} 40^{\prime} 23^{\prime \prime} \mathrm{W}$; alt. 350 m; 25 Jun 2007; P.H. Labiak \& F.B. Matos 3950 leg.; NY886643, UPCB9985.
Identification. Characterized by erect, underground stems, leaves with dichotomous to flabellate blades, and fertile segments (sporangiophores) pinnate, borne at the tips of the blades. Sporangia erect, oblong, with an apical annulus. The most similar species in the area is Actinostachys pennula (Sw.) Hook., which differs by subdigitate (vs pinnate) fertile segments.

Distribution and habitat. Neotropical. Terrestrial in forests, particularly at Morro do Mirante and Morro do Queimado.

Order Polypodiales Link (suborder Saccolomatineae Hovenkamp), Saccolomataceae Doweld

\section{Saccoloma elegans Kaulf.}

Figure 4I

Examined material. BRAZIL $\cdot 2$ specimens; Trilha do Zé Carlos; 2519'49"S, 048 39'14"W; alt. 30 m; 30 Apr. 2006; F.B. Matos \& G. Weiss 1131 leg.; MBM333138, UPCB9808.

Identification. Characterized by stout, woody, scaly, erect stems, petioles with omega-shaped vascular bundles (as seen in cross section), coriaceous leaves to $2 \mathrm{~m}$ long, 1-pinnate blades, submarginal sori, and indusia forming outward-facing conical cups. All other 1-pinnate species from the RPPN have different soral characters. Saccoloma brasiliense (C.Presl) Mett., which is the only other species of the genus occurring in the area, differs by 3-pinnate leaves.

Distribution and habitat. Neotropical. In Paraná, known only from RPPN Guaricica. Terrestrial in forests.

Order Polypodiales (suborder Lindsaeineae Lehtonen \& Tuomisto), Lindsaeaceae C.Presl ex M.R.Schomb.

\section{Lindsaea quadrangularis Raddi subsp. terminalis K.U.Kramer}

Figure 5A

Examined material. BRAZIL • 2 specimens; Trilha do Gervásio; 2514'39"S, 048³9'59"W, alt. 340 m; 27 May 2006; F.B. Matos \& M.C. Gomes 1179 leg.; MBM330188, UPCB6065 - 1 specimen; Trilha Esborracha Faisqueira; 2523'37"S, 048 40'52"W; alt. 15 m; 12 Jun. 2019; F.B. Matos et al. 2648 leg.; UPCB44506.

Identification. Characterized by creeping, scaly stems, the petioles reddish-brown to blackish or atropurpureous, subterete at the extreme base, otherwise quadrangular, the blades 2-pinnate, and the pinnules dimidiate. Sori submarginal, continuous along the upper margins, indusiate (indusia open outwardly). Differs from most Lindsaea species by the dark (vs stramineous) axes. Lindsaea divaricata Klotzsch also has dark axes, but differs by the presence of two lateral spreading wings on the adaxial side of rachises.

Distribution and habitat. Paraguay and Brazil. Terrestrial in forests. 


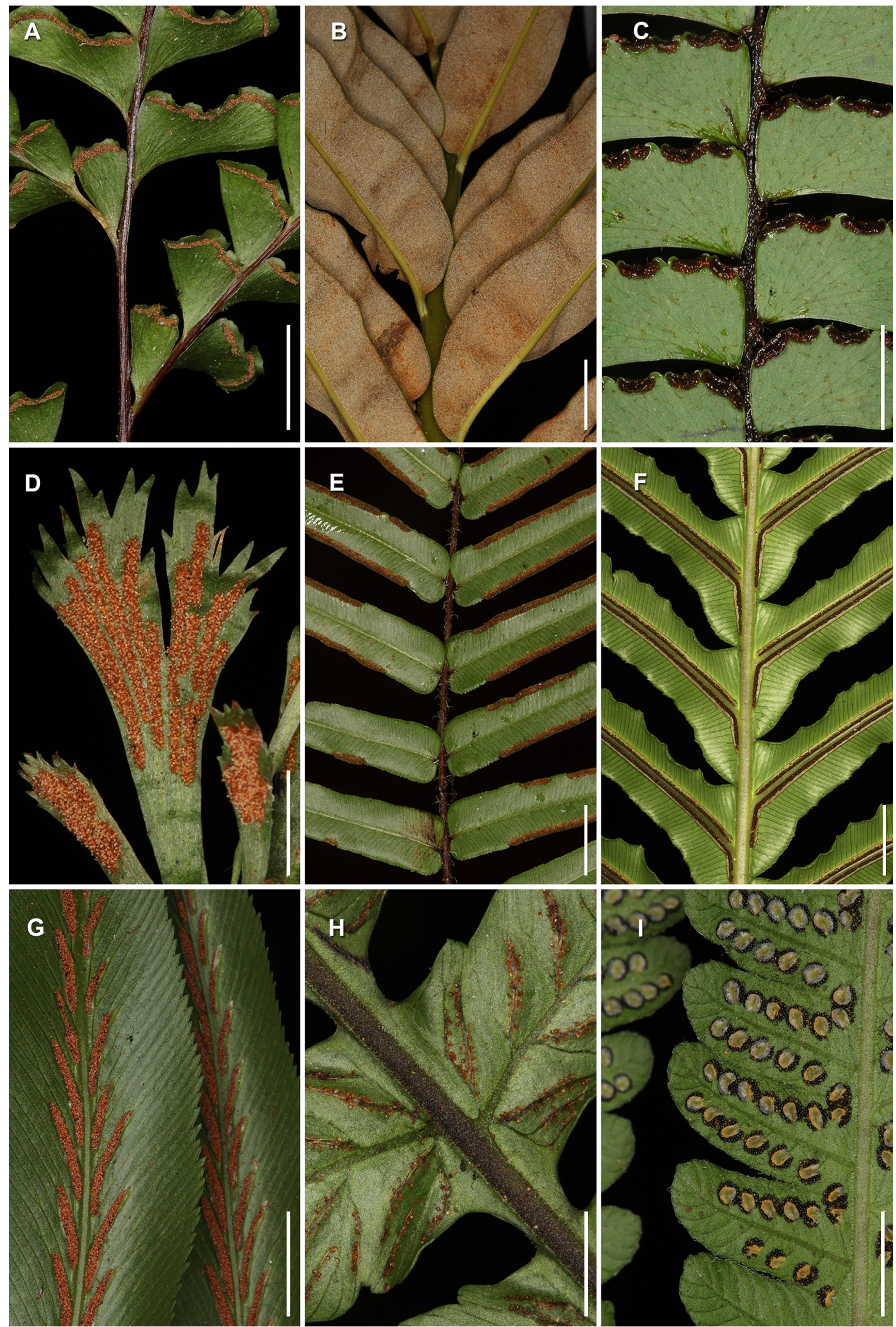

Figure 5. Some lycophytes and ferns from RPPN Guaricica. A. Lindsaea quadrangularis subsp. terminalis. B. Acrostichum danaeifolium. C. Adiantum humile. D. Hecistopteris pumila. E. Pteris vittata. F. Neoblechnum brasiliense. G. Asplenium serra. H. Diplazium ambiguum. I. Christella hispidula. (All photos by FBM). Scale bars: $0.5 \mathrm{~cm}(\mathbf{C}, \mathbf{D}, \mathbf{H}, \mathbf{I}) ; 1 \mathrm{~cm}(\mathbf{A}, \mathbf{B}, \mathbf{E}, \mathbf{F}, \mathbf{G})$. 
Order Polypodiales (suborder Pteridineae J.Prado \& Schuettp.), Pteridaceae E.D.M.Kirchn.

\section{Acrostichum danaeifolium Langsd. \& Fisch.}

Figures 3E, 5B

Examined material. BRAZIL - 2 specimens; Faisqueira; $25^{\circ} 22^{\prime} 14^{\prime \prime} \mathrm{S}, 048^{\circ} 39^{\prime} 56^{\prime \prime} \mathrm{W}$; alt. $15 \mathrm{~m} ; 7$ Oct. 2005; F.B. Matos \& P.B. Schwartsburd 855 leg.; MBM333063, UPCB120 • 5 specimens; Trilha do Queimado; $25^{\circ} 23^{\prime} 03^{\prime \prime} \mathrm{S}, 048^{\circ} 42^{\prime} 06^{\prime \prime} \mathrm{W}$; alt. $10 \mathrm{~m}$; $11 \mathrm{Jul} .2006$; F.B. Matos et al. 1211 leg.; CEPEC122106, MBM333064, NY886668, RB638223, UPCB123 • 1 specimen; Trilha Caixa d'Água; $25^{\circ} 23^{\prime} 19^{\prime \prime} \mathrm{S}, 048^{\circ} 40^{\prime} 05^{\prime \prime} \mathrm{W}$; alt. at sea level; 12 Jun. 2019; F.B. Matos et al. 2656 leg; UPCB44485 •

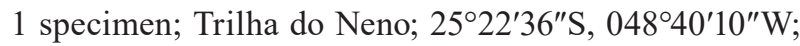
alt. 10 m; 28 Jun. 2007; P.H. Labiak et al. 3981 leg.; UPCB124.

Identification. Characterized by massive, erect stems, large fleshy roots, 1-pinnate, coriaceous leaves that are up to $5 \mathrm{~m}$ long, finely netted veins, and acrostichoid sori. It differs from $A$. aureum L., which was not found in the RPPN, by the presence of numerous, minute, erect hairs on the abaxial blade surfaces and leaves usually fertile for the entire length (vs fertile only in the distal 1/4-1/3).

Distribution and habitat. Neotropical. Terrestrial in brackish waters.

\section{Adiantum humile Kunze}

Figure 5C

Examined material. BRAZIL -2 specimens; Estrada Sede-Gervásio; $25^{\circ} 17^{\prime} 33^{\prime \prime} \mathrm{S}, \quad 048^{\circ} 42^{\prime} 15^{\prime \prime} \mathrm{W}$; alt. 30 m; 30 Apr. 2005; F.B. Matos \& M.O. Silva 547 leg.; MBM333067, UPCB287 • 2 specimens; Trilha da Guaricica; $25^{\circ} 19^{\prime} 13^{\prime \prime} \mathrm{S}, 048^{\circ} 42^{\prime} 04^{\prime \prime} \mathrm{W}$; alt. 20 m; 8 Oct. 2005; F.B. Matos \& P.B. Schwartsburd 877 leg.; MBM333066, UPCB286 • 1 specimen; Trilha da Pantera; 2518'02"S, 048 38'31"W; alt. 215 m; 29 May 2019; F.B. Matos et al. 2617 leg.; UPCB44503.

Identification. Characterized by short-creeping stems, 2-pinnate leaves with only 2-4 pairs of lateral pinnae, petioles and rachises with only one kind of scale (filiform subulate), pinnules abaxially glaucous and with long, septate, brown hairs, margins of sterile pinnules finely serrate, and glabrous indusia. Sori marginal, interrupted, the sporangia borne on the inner surface of the false indusium (not below it). Adiantum latifolium Lam. differs by long-creeping stems with leaves distant (vs approximate) from each other and pinnae abaxially glabrous. Adiantum terminatum Kunze ex Miq. differs by indusia bearing hairs.

Distribution and habitat. Neotropical. Terrestrial in forests.

\section{Hecistopteris pumila (Spreng.) J.Sm.}

Figure 5D

Examined material. BRAZIL • 2 specimens; Trilha do Gervásio; 2514'39"S, 048³9'59"W; alt. 50 m; 21 Dec.
2004; F.B. Matos \& P.H. Labiak 139 leg.; CEPEC122217, UPCB8526 • 2 specimens; Trilha do Corvo; 2519'39"S, $048^{\circ} 40^{\prime 2} 26^{\prime \prime} \mathrm{W}$; alt. 60 m; 6 Oct. 2005; F.B. Matos \& P.B. Schwartsburd 836 leg.; MBM333080, UPCB8547 • 1 specimen; Faisqueira; $25^{\circ} 22^{\prime} 14^{\prime \prime} \mathrm{S}, 048^{\circ} 39^{\prime} 56^{\prime \prime} \mathrm{W}$; alt. 20 m; 9 Oct. 2005; F.B. Matos \& P.B. Schwartsburd 882 leg.; UPCB8549 - 1 specimen; Trilha Porto do Rio Seco; 2524'19"S, 04840'54"W; alt. 15 m; 25 Jun. 2019; F.B. Matos \& V. Veiga 2662 leg.; UPCB44499 • 1 specimen; $25^{\circ} 14^{\prime} 28^{\prime \prime} \mathrm{S}, 048^{\circ} 40^{\prime} 23^{\prime \prime} \mathrm{W}$; alt. $200 \mathrm{~m}$; 25 Jun. 2007; P.H. Labiak \& F.B. Matos 3946 leg.; UPCB8550.

Identification. Characterized by the compact stems bearing long-creeping proliferous roots, very short or absent petioles, small (less than $4 \mathrm{~cm}$ long) flabellate leaves that are incised at the tip, and free, dichotomously forked veins. Sori elongate, without indusia, along the distal portion of veins. Not easily confused with any other fern from the area.

Distribution and habitat. Neotropical. Epiphytic in forests.

\section{Pteris vittata $\mathrm{L}$.}

Figure 5E

Examined material. BRAZIL -1 specimen; Fazenda Santa Olímpia; $25^{\circ} 16^{\prime} 45^{\prime \prime} \mathrm{S}, 048^{\circ} 43^{\prime} 57^{\prime \prime} \mathrm{W}$; alt. $15 \mathrm{~m} ; 27$ Aug. 2017; F.B. Matos \& J. Pontes 2605 leg.; UPCB44195 - 1 specimen; Estrada Cacatu-Guaraqueçaba, propriedade do Sr. Jandir; $25^{\circ} 18^{\prime} 38^{\prime \prime} \mathrm{S}, 048^{\circ} 41^{\prime} 01^{\prime \prime} \mathrm{W}$; alt. $15 \mathrm{~m}$; 29 May 2019; F.B. Matos et al. 2614 leg.; UPCB44189.

Identification. Characterized by the compact stems with yellowish scales, the densely scaly petioles, the 1-pinnate blades with linear pinnae, and the free veins. Sori linear and continuous on a submarginal connecting vein, protected by the reflexed margin of the blade (false indusium). Not easily confused with any other fern from the area.

Distribution and habitat. Native to eastern Asia, frequently introduced and naturalized in the New World tropics and subtropics. Epipetric in sunny and open places, particularly on masonry walls and concrete structures. Also recorded as terrestrial outside the RPPN, usually in calcareous soil.

Order Polypodiales (suborder Aspleniineae H.Schneid. \& C.J.Rothf.), Blechnaceae Newman

\section{Neoblechnum brasiliense (Desv.) Gasper \& V.A.O.Dittrich}

Figure $5 \mathrm{~F}$

Examined material. BRAZIL • 1 specimen; Trilha das Arapongas; $25^{\circ} 18^{\prime} 11^{\prime \prime} \mathrm{S}, 048^{\circ} 41^{\prime} 10^{\prime \prime} \mathrm{W}$; alt. $20 \mathrm{~m}$; 1 Mar. 2005; F.B. Matos \& U. Ferreira 472 leg.; UPCB2223 • 1 specimen; Trilha do Mirante; $25^{\circ} 14^{\prime} 25^{\prime \prime} \mathrm{S}, 048^{\circ} 39^{\prime} 55^{\prime \prime} \mathrm{W}$; alt. 100 m; 12 Sep. 2008; F.B. Matos et al. 1595 leg.; UPCB2238 - 1 specimen; Trilha Caixa d'Água; $25^{\circ} 23^{\prime} 19^{\prime \prime} \mathrm{S}, 048^{\circ} 40^{\prime} 05^{\prime \prime} \mathrm{W}$; alt. at sea level; 12 Jun. 2019; F.B. Matos et al. 2633 leg.; UPCB43860. 
Identification. Characterized by the erect, trunk-like stems clothed with black scales, the monomorphic leaves with pinnate to pinnatisect blades that are gradually reduced basally, and the linear to linear-lanceolate pinnae that are adnate to rachises and serrulate throughout. Sori linear and parallel to the midrib, indusiate (indusia open inwardly). Not easily confused with any other fern from the area.

Distribution and habitat. Neotropical. Terrestrial in degraded areas.

Order Polypodiales (suborder Aspleniineae), Aspleniaceae Newman

\section{Asplenium serra Langsd. \& Fisch.}

Figure $5 \mathrm{G}$

Examined material. BRAZIL - 3 specimens; Porto do Cachaça; $25^{\circ} 20^{\prime} 27^{\prime \prime} \mathrm{S}, 048^{\circ} 41^{\prime} 47^{\prime \prime} \mathrm{W}$; alt. $10 \mathrm{~m}$; 12 Jul. 2006; F.B. Matos et al. 1227a leg.; CEPEC118585, NY886670, RB642392 • 1 specimen; Morro do Queimado; $25^{\circ} 23^{\prime} 10^{\prime \prime} \mathrm{S}, 048^{\circ} 42^{\prime} 19^{\prime \prime} \mathrm{W}$; alt. $300 \mathrm{~m}$; 12 Jun. 2019; F.B. Matos et al. 2640 leg.; UPCB44417.

Identification. Characterized by short- to long-creeping stems with castaneous, lanceolate, clathrate scales, 1-pinnate leaves, and lanceolate pinnae with margins serrate to biserrate. Sori elongate, oriented obliquely to the midrib, indusiate (indusia open inwardly). The most similar species in the area is Asplenium incurvatum Fée, which differs by blackish, linear stem scales with acute to acuminate (vs filiform-tipped) apices.

Distribution and habitat. Neotropical. Epiphytic, occasionally terrestrial in forests.

Order Polypodiales (suborder Aspleniineae), Athyriaceae Alston

\section{Diplazium ambiguum Raddi}

Figure $5 \mathrm{H}$

Examined material. BRAZIL - 3 specimens; Trilha Rio do Ferro; $25^{\circ} 18^{\prime} 07^{\prime \prime} \mathrm{S}, 048^{\circ} 40^{\prime} 49^{\prime \prime} \mathrm{W}$; alt. $50 \mathrm{~m}$; 16 Jan. 2005; F.B. Matos 210 leg.; MBM333055, RB642975, UPCB2884 • 2 specimens; Trilha dos Fornos; 2517'38's, $048^{\circ} 39^{\prime} 25^{\prime \prime} \mathrm{W}$; alt. 100 m; 2 Mar. 2005; F.B. Matos \& U. Ferreira 477 leg.; RB642992, UPCB2885 • 3 specimens; Trilha do Corvo; 2519'39"S, 048 40'26"W; alt. $60 \mathrm{~m}$; 6 Oct. 2005; F.B. Matos \& P.B. Schwartsburd 831 leg.; MBM333056, RB642979, UPCB2890 • 2 specimens; Trilha do Gervásio; 2514'39"S, 048³9'59"W; alt. 100 m; 27 May 2006; F.B. Matos 1183 leg.; MBM333057, UPCB2889 • 1 specimen; Trilha do Mirante; 2514'25"S, $048^{\circ} 39^{\prime} 55^{\prime \prime} \mathrm{W}$; alt. 200 m; 12 Sep. 2008; F.B. Matos et al. 1601 leg.; UPCB2950 • 1 specimen; Trilha da Pantera; $25^{\circ} 18^{\prime} 02^{\prime \prime} \mathrm{S}, 048^{\circ} 38^{\prime} 31^{\prime \prime} \mathrm{W}$; alt. $240 \mathrm{~m}$; 29 May 2019; F.B. Matos et al. 2619 leg.; UPCB44395 - 1 specimen; 2519'30"S, 04846'30"W; alt. 200 m; 1 Sep. 2006; M.C. Gomes et al. 14 leg.; UPCB2887 • 1 specimen; 2519'30"S, $048^{\circ} 46^{\prime} 30^{\prime \prime} \mathrm{W}$; alt. 100 m; 1 Sep. 2006; M.C. Gomes et al.
32 leg.; UPCB2942 • 1 specimen; same collection data as for preceding; M.C. Gomes et al. 36 leg.; UPCB2900.

Identification. Characterized by erect, trunk-like stems, 2-pinnate-pinnatifid leaves gradually tapering to a pinnatifid apex, and glabrous abaxial blade surfaces. Sori linear, indusiate, usually paired back to back along a single vein. It is often confused with Diplazium asplenioides (Kunze) C.Presl, which was not recorded in the area and differs by the presence of numerous hairs on abaxial blade surfaces and indusia.

Distribution and habitat. Widely distributed in South America. Terrestrial in forests.

Order Polypodiales (suborder Aspleniineae), Thelypteridaceae Ching ex Pic.Serm.

\section{Christella hispidula (Decne.) Holttum \\ Figure 5I}

Examined material. BRAZIL • 2 specimens; Fazenda Rincão; $25^{\circ} 18^{\prime} 53^{\prime \prime} \mathrm{S}, 048^{\circ} 41^{\prime} 46^{\prime \prime} \mathrm{W}$; alt. $30 \mathrm{~m}$; 1 Mar. 2005; F.B. Matos \& U. Ferreira 466 leg.; MBM333033, UPCB10243 - 2 specimens; Estrada Sede-Gervásio; $25^{\circ} 17^{\prime} 33^{\prime \prime} \mathrm{S}, 048^{\circ} 42^{\prime} 15^{\prime \prime} \mathrm{W}$; alt. 30 m; 30 Apr. 2005; F.B. Matos \& M.O. Silva 539 leg.; MBM333034, UPCB10249 - 2 specimens; same collection data as for preceding; F.B. Matos \& M.O. Silva 543 leg.; MBM333035, UPCB10248 - 2 specimens; Faisqueira; 2522'14"S, 048 39'56"W; alt. $15 \mathrm{~m} ; 7$ Oct. 2005; F.B. Matos \& P.B. Schwartsburd 858 leg.; MBM333036, UPCB10271 • 1 specimen; Trilha da Guaricica; $25^{\circ} 19^{\prime} 13^{\prime \prime S}, 048^{\circ} 42^{\prime} 04^{\prime \prime} \mathrm{W}$; alt. 20 m; 8 Oct. 2005; F.B. Matos \& P.B. Schwartsburd 867 leg.; UPCB10268 - 2 specimens; Trilha dos Pinheiros; $25^{\circ} 20^{\prime} 05^{\prime \prime} \mathrm{S}, 048^{\circ} 40^{\prime} 54^{\prime \prime} \mathrm{W}$; alt. 30 m; 30 Apr. 2006; F.B. Matos \& G. Weiss 1124 leg.; MBM333037, UPCB10269 - 1 specimen; Fazenda Santa Olímpia; 2516'45"S, 04843'57"W; alt. 15 m; 27 Aug. 2017; F.B. Matos \& J. Pontes 2604 leg.; UPCB44196 • 1 specimen; Trilha da Rede; $25^{\circ} 17^{\prime} 49^{\prime \prime} \mathrm{S}, 048^{\circ} 40^{\prime} 23^{\prime \prime} \mathrm{W}$; alt. 25 m; 25 Jun. 2019; F.B. Matos \& V. Veiga 2673 leg.; UPCB44390.

Identification. Characterized by short-creeping stems, 1-pinnate-pinnatifid leaves, the lowermost veins of adjacent segments uniting below sinuses with an excurrent vein to the sinuses, and the indument abaxially of numerous, unbranched, acicular hairs. Sori round, with large and hairy indusia. It is often confused with Christella dentata (Forssk.) Brownsey \& Jermy, which differs by uniformly short hairs (0.1-0.2 $\mathrm{mm}$ long) on the costae abaxially (vs hairs of variable length, $0.3-0.8 \mathrm{~mm}$ long, in C. hispidula).

Distribution and habitat. Pantropical, introduced and naturalized in the Americas, from SE USA to Uruguay. Terrestrial in degraded areas.

\section{Goniopteris paranaensis (Salino) Salino \& T.E.Almeida \\ Figure 6A}

Examined material. BRAZIL - 2 specimens; Trilha 


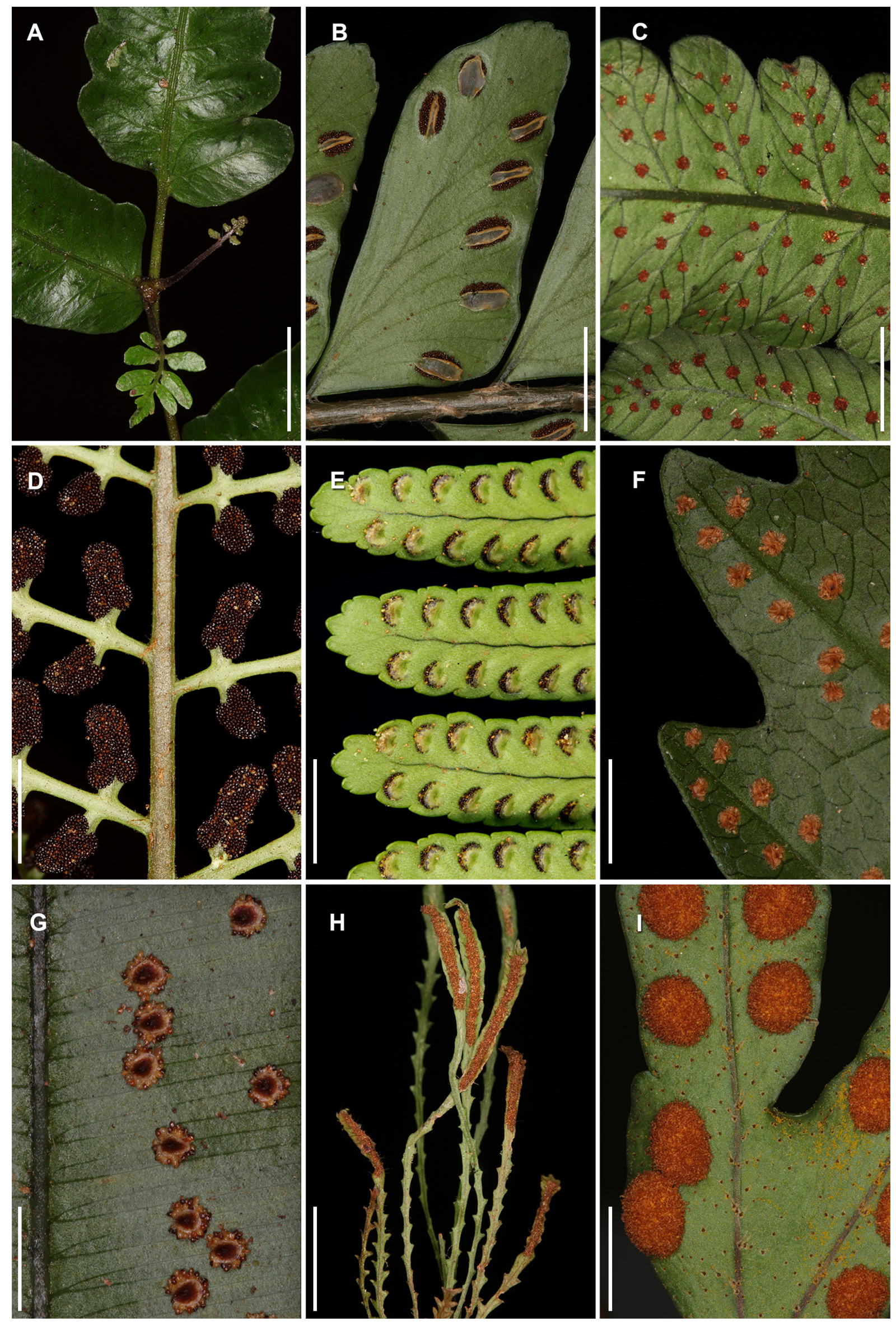

Figure 6. Some lycophytes and ferns from RPPN Guaricica. A. Goniopteris paranaensis. B. Didymochlaena truncatula. C. Ctenitis nervata. D. Polybotrya cylindrica. E. Nephrolepis exaltata. F. Tectaria pilosa. G. Oleandra australis, a species known only from RPPN Guaricica. H. Cochlidium serrulatum. I. Pleopeltis pleopeltifolia. (All photos by FBM; except D and E, by Nathan Smith). Scale bars: $0.5 \mathrm{~cm}(\mathbf{B}, \mathbf{D}, \mathbf{G}, \mathbf{I}) ; 1 \mathrm{~cm}$ $(A, C, E, F, H)$. 


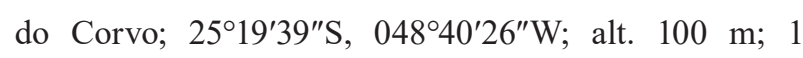
May 2005; F.B. Matos et al. 575 leg.; MBM333043, UPCB10347 • 2 specimens; 2519'39"S, 048 40'26"W; alt. 100 m; 6 Oct. 2005; F.B. Matos \& P.B. Schwartsburd 823 leg.; RB706145, UPCB10352 • 4 specimens; Trilha dos Pinheiros; $25^{\circ} 20^{\prime} 05^{\prime \prime} \mathrm{S}, 048^{\circ} 40^{\prime} 54^{\prime \prime} \mathrm{W}$; alt. $130 \mathrm{~m} ; 6$ Oct. 2005; F.B. Matos \& P.B. Schwartsburd 844 leg.; CEPEC122246, MBM333044; RB706149; UPCB10348

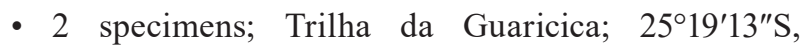
$048^{\circ} 42^{\prime} 04^{\prime \prime} \mathrm{W}$; alt. 20 m; 8 Oct. 2005; F.B. Matos \& P.B. Schwartsburd 868 leg.; MBM333045, UPCB10345 • 1 specimen; Trilha do Mirante; $25^{\circ} 14^{\prime} 25^{\prime \prime} \mathrm{S}, 048^{\circ} 39^{\prime} 55^{\prime \prime} \mathrm{W}$; alt. 200 m; 12 Sep. 2008; F.B. Matos et al. 1606 leg.; UPCB10350 - 1 specimen; Morro do Queimado; 2523'10"S, 04842'19"W; alt. 150 m; 12 Jun. 2019; F.B. Matos et al. 2647 leg.; UPCB44496 • 1 specimen; $25^{\circ} 19^{\prime} 30^{\prime \prime} \mathrm{S}, 048^{\circ} 46^{\prime} 30^{\prime \prime} \mathrm{W}$; alt. 100 m; 1 Sep. 2006; M.C. Gomes et al. 31 leg.; UPCB10349.

Identification. Characterized by short-creeping stems, 1-pinnate-pinnatifid leaves, conform blade apices, free veins, the lowermost veins of adjacent segments connivent at the sinuses, proliferous buds (sometimes plantlets) present distally on the blades, and indumentum typically of minute forked or stellate hairs. Sori round, indusiate, the indusia densely pilose. The most similar species in the area are Goniopteris vivipara (Raddi) Brade and G. lugubris (Mett.) Brade. The first differs by less incised pinnae (nearly entire vs incised ca $1 / 3-2 / 3$ ), whereas the second differs by pinnatifid blade apices (vs conform blade apices in G. paranaensis).

Distribution and habitat. Endemic to Brazil, occurring only in São Paulo and Paraná. Terrestrial in forests.

Order Polypodiales (suborder Polypodiineae Dumort.), Didymochlaenaceae Ching ex Li Bing Zhang \& Liang Zhang

\section{Didymochlaena truncatula (Sw.) J.Sm.}

Figure 6B

Examined material. BRAZIL • 1 specimen; Trilha da Estrada; $25^{\circ} 17^{\prime} 17^{\prime \prime} \mathrm{S}, 048^{\circ} 38^{\prime} 40^{\prime \prime} \mathrm{W}$; alt. $100 \mathrm{~m}$; 2 Mar. 2005; F.B. Matos \& U. Ferreira 481 leg.; UPCB3716 • 1 specimen; Trilha do Meio; $25^{\circ} 14^{\prime} 38^{\prime \prime} \mathrm{S}, 048^{\circ} 39^{\prime} 13^{\prime \prime} \mathrm{W}$; alt. 200 m; 28 May 2006; F.B. Matos \& M.C. Gomes 1188 leg.; UPCB3717 • 1 specimen; Trilha Porto do Rio Seco; $25^{\circ} 24^{\prime} 19^{\prime \prime} \mathrm{S}, 048^{\circ} 40^{\prime} 54^{\prime \prime} \mathrm{W}$; alt. 15 m; 25 Jun. 2019; F.B. Matos \& V. Veiga 2668 leg.; UPCB44501.

Identification. Characterized by massive, scaly, erect stems, 2-pinnate leaves up to $1.5 \mathrm{~m}$ long, subdimidiate pinnules that are sessile and obtuse, basal acroscopic pinnules overlapping the rachis, and minute spine-like projections on the upper surface of the junctures of the rachis, costae, and costules. Sori elongate, on both sides of the vein, indusia attached to vein along medial line. Not easily confused with any other fern from the area.

Distribution and habitat. Pantropical. Terrestrial in forests.
Order Polypodiales (suborder Polypodiineae), Dryopteridaceae Herter

\section{Ctenitis nervata (Fée) R.S.Viveros \& Salino}

Figure 6C

Examined material. BRAZIL - 1 specimen; Trilha dos Pinheiros; $25^{\circ} 20^{\prime} 05^{\prime \prime} \mathrm{S}, 048^{\circ} 40^{\prime} 54^{\prime \prime} \mathrm{W}$; alt. $130 \mathrm{~m} ; 6$ Oct. 2005; F.B. Matos \& P.B. Schwartsburd 846 leg.; UPCB3858 - 1 specimen; Trilha do Gervásio; $25^{\circ} 14^{\prime} 39^{\prime \prime} \mathrm{S}$, $048^{\circ} 39^{\prime} 59^{\prime \prime} \mathrm{W}$; alt. 100 m; 28 Jul. 2006; F.B. Matos et al. 1234 leg.; UPCB3868 • 1 specimen; same collection data as for preceding; 7 Sep. 2006; F.B. Matos et al. 1242 leg.; UPCB3862 • 1 specimen; Trilha do Mirante; $25^{\circ} 14^{\prime} 25^{\prime \prime} \mathrm{S}$, 048 39'55"W; alt. 100 m; 26 Apr. 2019; F.B. Matos et al. 2612 leg.; UPCB44191 • 1 specimen; Morro do Quei-

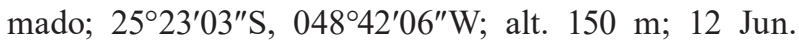
2019; F.B. Matos et al. 2644 leg.; UPCB44492.

Identification. Formerly known as Ctenitis pedicellata (Christ) Copel., which is now a synonym (Viveros et al. 2018). It is characterized by erect stems with dark brown to blackish scales, 1-pinnate-pinnatifid leaves, and the pinnae incised ca $1 / 2-2 / 3$ the distance between segment apices and costae. Sori round, lacking indusia. Ctenitis submarginalis (Langsd. \& Fisch.) Ching is the only other species of the genus in the area, differing by orangish stem scales and pinnae incised more than 3/4 the distance between segment apices and costae.

Distribution and habitat. Bolivia and Brazil. Terrestrial in forests.

\section{Polybotrya cylindrica Kaulf. \\ Figure 6D}

Examined material. BRAZIL - 1 specimen; Trilha do Bezerra; 2519'25"S, 048 $41^{\prime} 26^{\prime \prime} \mathrm{W}$; 10 May 2003; M. Borgo et al. 2497 leg.; UPCB4735 - 1 specimen; Trilha do Gervásio; 25¹4'39"S, 048³9'59"W; alt. 50 m; 21 Dec. 2004; F.B. Matos \& P.H. Labiak 141 leg.; UPCB4738 - 1 specimen; Trilha dos Pinheiros; $25^{\circ} 20^{\prime} 05^{\prime \prime} \mathrm{S}, 048^{\circ} 40^{\prime} 54^{\prime \prime} \mathrm{W}$; alt. $60 \mathrm{~m}$; 6 Oct. 2005; F.B. Matos \& P.B. Schwartsburd 840 leg.; UPCB4739 • 1 specimen; Trilha do Mirante; $25^{\circ} 14^{\prime} 25^{\prime \prime} \mathrm{S}, 048^{\circ} 39^{\prime} 55^{\prime \prime} \mathrm{W}$; alt. 200 m; 12 Sep. 2008; F.B. Matos et al. 1604 leg.; UPCB4730 - 1 specimen; Trilha da Rede; $25^{\circ} 17^{\prime} 49^{\prime \prime} \mathrm{S}$, $048^{\circ} 40^{\prime 2} 23^{\prime \prime}$; alt. 300 m; 26 Aug. 2017; F.B. Matos et al. 2560 leg.; UPCB44005 • 1 specimen; Trilha do Gervásio; 2514'39"S, 048 39'59"W; alt. 100 m; 27 Aug. 2017; F.B. Matos et al. 2578 leg.; UPCB44068 1 specimen; Trilha da Pantera; $25^{\circ} 18^{\prime} 02^{\prime \prime S}, 048^{\circ} 38^{\prime} 31^{\prime \prime} \mathrm{W}$; alt. 215 m; 29 May 2019; F.B. Matos et al. 2616 leg.; UPCB44493 • 1 specimen; Trilha Caixa d'Água; $25^{\circ} 23^{\prime} 19^{\prime \prime} \mathrm{S}, 048^{\circ} 40^{\prime} 05^{\prime \prime} \mathrm{W}$; alt. at sea level; 12 Jun. 2019; F.B. Matos et al. 2653 leg.; UPCB44487 - 1 specimen; Fazenda da SPVS; $25^{\circ} 18^{\prime} 53^{\prime \prime} \mathrm{S}, 048^{\circ} 41^{\prime} 46^{\prime \prime} \mathrm{W}$; alt. 40 m; 20 Oct. 2003; P.H. Labiak \& R. Goldenberg 3013 leg.; UPCB4713 • 1 speci-

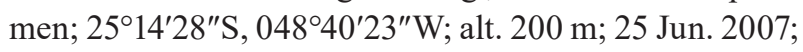
P.H. Labiak \& F.B. Matos 3952 leg.; UPCB4741.

Identification. Characterized by long-creeping stems 
clothed with dark brown scales, ovate to deltate, usually 3-pinnate-pinnatifid leaves, the apex pinnatifid, sterile and fertile leaves strongly dimorphic, and free veins. Sori acrostichoid, indusia absent. It is one of the most abundant ferns in the RPPN. Not easily confused with any other species from the area.

Distribution and habitat. Endemic to Brazil, from Espírito Santo and Minas Gerais to Rio Grande do Sul. Hemiepiphytic in forests.

Order Polypodiales (suborder Polypodiineae), Nephrolepidaceae Pic.Serm.

\section{Nephrolepis exaltata (L.) Schott}

Figure 6E

Examined material. BRAZIL -2 specimens; Trilha do Zé Carlos; 2519'49"S, 048³9'14"W; alt. 30 m; 30 Apr. 2006; F.B. Matos \& G. Weiss 1133 leg.; MBM333010; UPCB6288.

Identification. Characterized by suberect, stoloniferous stems, spreading petiolar scales, 1-pinnate leaves, linear blades strongly reduced towards the base, the pinnae nearly sessile and articulated to the rachis, and free, hydathodous veins. Sori round to elongated, indusia reniform, with wide U-shaped sinus. The other two species of the genus in the area differ by basal petiolar scales shining (vs dull), laminar base truncate or moderately reduced (vs strongly reduced), and indusia with narrow sinus (vs wide sinus in N. exaltata).

Distribution and habitat. Native range uncertain, widely cultivated worldwide (introduced in the Old World tropics). Terrestrial in disturbed areas.

Order Polypodiales (suborder Polypodiineae), Tectariaceae Panigrahi

\section{Tectaria pilosa (Fée) R.C.Moran}

Figures 3D, 6F

Examined material. BRAZIL -2 specimens; Trilha da Estrada; $25^{\circ} 17^{\prime} 17^{\prime \prime} \mathrm{S}, 048^{\circ} 38^{\prime} 40^{\prime \prime} \mathrm{W}$; alt. $100 \mathrm{~m}$; 2 Mar. 2005; F.B. Matos \& U. Ferreira 490 leg.; MBM333150, UPCB9903 - 1 specimen; Trilha Porto do Rio Seco; 2524'19"S, 048 40'54"W; alt. 15 m; 25 Jun. 2019; F.B. Matos \& V. Veiga 2665 leg.; UPCB44495.

Identification. Characterized by erect stems, 1-pinnate leaves with 1-4 pinna pairs, basal pinnae with a single lobe on the basal basiscopic side, blades puberulent on both surfaces, and veins reticulate, the areoles typically with included veinlets. Sori round, indusiate. Differs from the other species of the genus by the presence of hairs on the blade and less pinna pairs (1-4 vs 4-12).

Distribution and habitat. Neotropical. Terrestrial in forests, usually growing on shell middens.

Order Polypodiales (suborder Polypodiineae), Oleandraceae Ching ex Pic.Serm.

\section{Oleandra australis Schwartsb. \& J.Prado}

Figures 3B, 6G

Examined material. BRAZIL - 1 specimen; Morro do Queimado; $25^{\circ} 23^{\prime} 10^{\prime \prime} \mathrm{S}, 048^{\circ} 42^{\prime} 19^{\prime \prime} \mathrm{W}$; alt. $300 \mathrm{~m} ; 12$ Jun. 2019; F.B. Matos et al. 2635 leg.; UPCB43863 • 3 specimens; $25^{\circ} 14^{\prime} 28^{\prime \prime} \mathrm{S}, 048^{\circ} 40^{\prime} 23^{\prime \prime} \mathrm{W}$; alt. $350 \mathrm{~m}$; 25 Jun. 2007; P.H. Labiak \& F.B. Matos 3941 leg.; MBM393020, NY886641, UPCB6338.

Identification. Characterized by long-creeping stems with bicolorous peltate scales, stipes articulate to phyllopodia, undivided, elliptical laminae, and free veins. Sori round, indusiate, the indusia bicolorous and attached at a narrow sinus. It could be confused with some species of Elaphoglossum Schott ex J.Sm., which differ by nonpeltate stem scales, ascending veins (vs veins nearly perpendicular to the midrib in Oleandra), and acrostichoid sori.

Distribution and habitat. Endemic to RPPN Guaricica; known from only two collections. Terrestrial, hanging on steep slopes in montane forests.

Order Polypodiales (suborder Polypodiineae), Polypodiaceae J.Presl \& C.Presl

\section{Cochlidium serrulatum (Sw.) L.E.Bishop}

Figures $6 \mathrm{H}$

Examined material. BRAZIL 11 specimen; Trilha do Gervásio; 25¹4'39"S, 048³9'59"W; alt. 50 m; 21 Dec. 2004; F.B. Matos \& P.H. Labiak 138 leg.; UPCB6887 • 1 specimen; Trilha Esborracha Faisqueira; 25 23'37"S, $048^{\circ} 40^{\prime} 52^{\prime \prime} \mathrm{W}$; alt. $15 \mathrm{~m}$; 12 Jun. 2019; F.B. Matos et al. 2649 leg.; UPCB44481 - 1 specimen; 2514'28"S, 048 40'23"W; alt. 200 m; 25 Jun. 2007; P.H. Labiak \& F.B. Matos 3947 leg.; UPCB6885.

Identification. Characterized by erect stems, and small $(10-20 \times 2-3 \mathrm{~mm})$, deeply pinnatifid leaves. Sori on lateral veins, coalescing at maturity into a coenosorus on each side of the midrib. Not easily confused with any other fern from the area.

Distribution and habitat. Pantropical. Epiphytic in forests.

\section{Pleopeltis pleopeltifolia (Raddi) Alston}

Figure 6I

Examined material. BRAZIL $\bullet 2$ specimens; Trilha da Estrada; $25^{\circ} 17^{\prime} 17^{\prime \prime} \mathrm{S}, 048^{\circ} 38^{\prime} 40^{\prime \prime} \mathrm{W}$; alt. $100 \mathrm{~m}$; 2 Mar. 2005; F.B. Matos \& U. Ferreira 491 leg.; MBM333128, UPCB7748 - 2 specimens; Trilha do Gervásio; 2514'39"S, 048 39'59"W; 27 May 2006; F.B. Matos \& M.C. Gomes 1187 leg.; MBM333127, UPCB7861 - 1 specimen; Fazenda Santa Olímpia; 2516 $45^{\prime \prime} \mathrm{S}$, 04843'57"W; alt. 15 m; 27 Aug. 2017; F.B. Matos \& J. Pontes 2606 leg.; UPCB44194 • 1 specimen; 25¹9’30"S, $048^{\circ} 46^{\prime} 30^{\prime \prime} \mathrm{W}$; alt. $100 \mathrm{~m}$; 1 Sep. 2006; M.C. Gomes et al. 44 leg.; UPCB7858 - 1 specimen; Trilha do Neno; $25^{\circ} 22^{\prime} 36^{\prime \prime} \mathrm{S}, 048^{\circ} 40^{\prime} 10^{\prime \prime} \mathrm{W}$; alt. 10 m; 28 Jun. 2007; P.H. Labiak et al. 3967 leg.; UPCB7819. 
Identification. Characterized by short-creeping stems with blackish, peltate, linear-lanceolate scales, deeply pinnatisect blades with numerous, peltate, ovate to lanceolate scales, and obscured anastomosing veins. Sori round, with peltate soral scales, without indusia. It is one of the most common species in the RPPN. Not easily confused with any other fern from the area.

Distribution and habitat. Paraguay, Argentina, Uruguay, and Brazil. Epiphytic in a variety of habitats, also observed on masonry walls.

\section{Discussion}

The species found in our study correspond to approximately $47 \%$ or $41.5 \%$ of the pteridophytes recorded for the state of Paraná in previous studies (Kaehler et al. 2014; Prado et al. 2015, respectively). Our checklist fills a gap between two other recent floristic studies, those for Ilha do Mel (Salino et al. 2005, with 114 spp.) and Pico Paraná State Park (Pereira and Labiak 2018, with $142 \mathrm{spp}$.), and nearly completes an elevational gradient from sea level to the top of the highest mountain in southern Brazil (i.e., Pico Paraná at about 1890 m). Taken together, these three studies give us a good picture of the pteridophyte flora of the Atlantic rainforest in Paraná. With 204 species, RPPN Guaricica has more ferns and lycophytes than any other area in that state. Its richness is followed by the Guartelá State Park (163 spp., Michelon and Labiak 2013), Vila Velha State Park (152 spp., Schwartsburd and Labiak 2007), Pico Paraná State Park (142 spp., Pereira and Labiak 2018), Ilha do Mel (114 spp., Salino et al. 2005), and Iguaçu National Park (101 spp., Lautert et al. 2015). Some mountainous areas at Serra do Mar, however, will probably show a higher number of species when studied more thoroughly. We consider the following areas as potential record breakers, based on their partial inventories: Marumbi State Park (81 spp. in a 1-ha plot at $630 \mathrm{~m}$, Dittrich et al. 2005), Parque Nacional Saint-Hilaire/Lange (74 epiphytic spp., Blum et al. 2011), and Pico Paraná State Park (142 spp. above 1000 $\mathrm{m}$ in elevation, Pereira and Labiak 2018). Among these areas, the pteridophyte flora of RPPN Guaricica is most similar to that of Ilha do Mel (Salino et al. 2005), a continental island with ca 2900 ha and elevations from sea level to $148 \mathrm{~m}$ (Britez and Marques 2005). There are only 12 species from this island that have not been found at RPPN Guaricica: Actinostachys subtrijuga (Mart.) C.Presl, Adiantum diogoanum Glaz. ex Baker, Asplenium lacinulatum Schrad., Ctenitis paranaensis (C.Chr.) Lellinger [as C. falciculata (Raddi) Ching], Pseudolycopodiella meridionalis (Underw. \& Lloyd) Holub [as Lycopodiella caroliniana (L.) Pic. Serm.], Microgramma
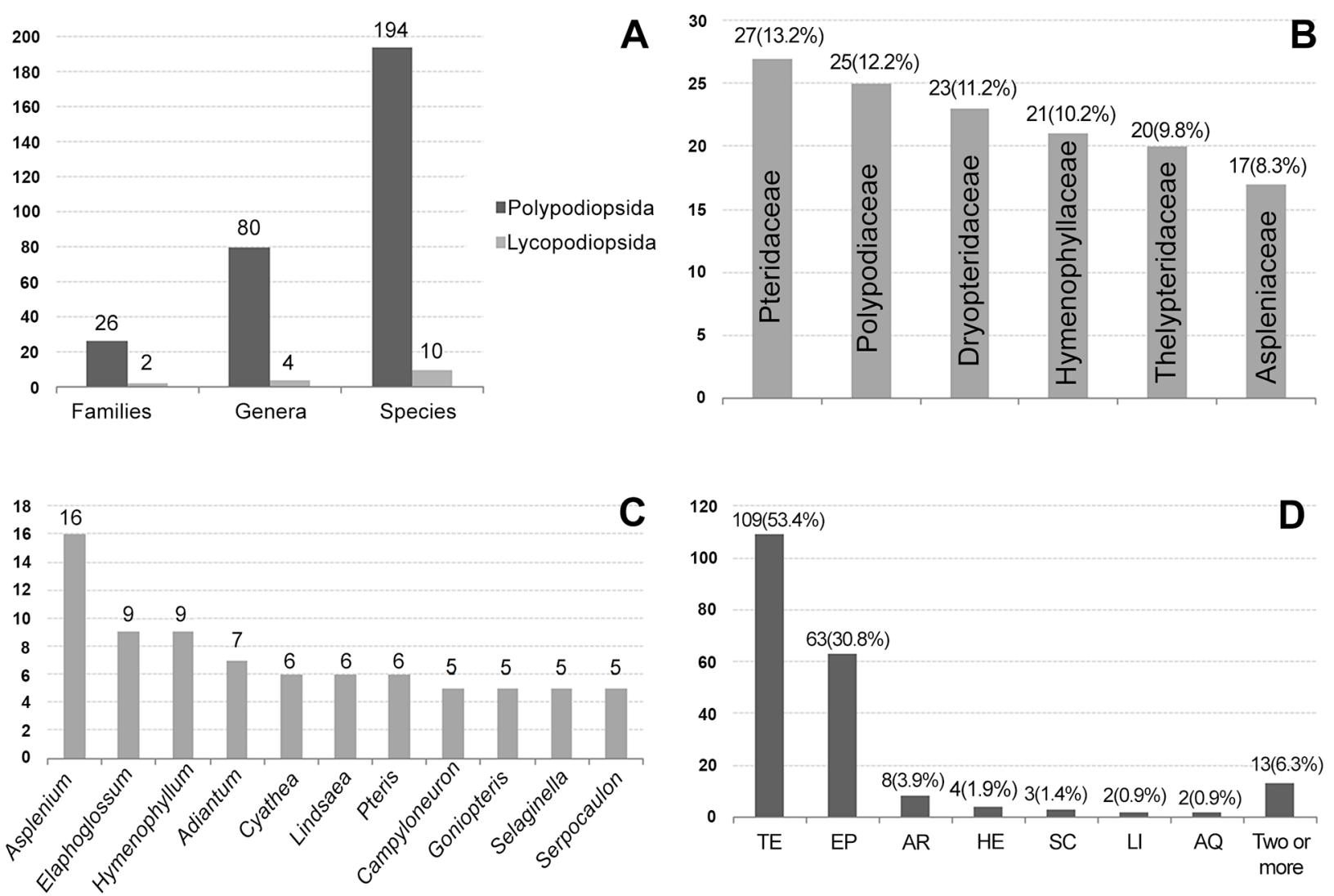

Figure 7. Numbers of ferns and lycophytes in Reserva Natural Guaricica by classes, families, genera, and growth forms. A. Proportion of families, genera, and species of Lycopodiopsida and Polypodiopsida. B. The six most diverse families of ferns (percentage of species in brackets). C. The 11 most diverse genera of ferns and lycophytes. D. Number of species per growth forms (percentage of species in brackets). The growth forms are classified as terrestrial (TE), epiphyte (EP), arborescent (AR), hemiepiphyte (HE), scandent (SC), litophytic $(\mathrm{LI})$, and aquatic $(\mathrm{AQ})$. 
geminata (Schrad.) R.M.Tryon \& A.F.Tryon, Osmundastrum cinnamomeum (L.) C.Presl (as Osmunda cinnamomea L.), Pecluma paradiseae (Langsd. \& Fisch.) M.G.Price, Pleopeltis furcata (L.) A.R.Sm. [as Dicranoglossum furcatum (L.) J.Sm.], Pteris brasiliensis Raddi, Pteris denticulata Sw., and Schizaea fluminensis Miers ex J.W.Sturm. The specimens cited as Hymenophyllum vestitum (C.Presl) Bosch for Ilha do Mel were wrongly identified and refer instead to H. hirsutum (L.) Sw. (F. Gonzatti, pers. comm.). Given the geographical proximity and the floristic similarities between the two areas, it is likely that all species from Ilha do Mel will eventually be found at RPPN Guaricica as more collections become available.

At a national level, there are very few studies comprising more species of ferns and lycophytes. These are Mazziero et al. (2018) (251 species), Salino and Almeida (2008) (212 species), and Souza et al. (2012) (209 species). It is worth mentioning, however, that these three studies were conducted in much larger areas $(35,700$, 150,000 , and 10,000 ha, respectively) with a wider elevational range $(0-1700 \mathrm{~m})$.

One aspect of the diversity of pteridophytes in RPPN Guaricica is the variation in size, from tiny filmy ferns [Didymoglossum angustifrons and D. hymenoides (Hedw.) Copel.] and floating aquatic ferns (Azolla filiculoides Lam.), with leaves smaller than $0.5 \mathrm{~cm}$ long, to towering tree ferns whose trunks reach $15 \mathrm{~m}$ tall bearing leaves ca $4 \mathrm{~m}$ long [e.g. Alsophila sternbergii (Sternb.) D.S.Conant]. Even longer are the vine-like leaves of Lygodium volubile Sw. and Salpichlaena volubilis (Kaulf.) J.Sm. These two species have creeping rhizomes, but their leaves can grow up to $30 \mathrm{~m}$ long, having a twinning rachis that uses the surrounding vegetation for support (R. Moran, pers. comm.). Another aspect, perhaps not so readily seen, is the high phylogenetic diversity found at the RPPN. Nearly all of the main clades proposed by the PPG 1 (2016) are present in the area. The only exceptions are Equisetales, Isoetales, and Psilotales.

According to the literature (e.g. Kress 1986; Benzing 1990, 2012; Schuettpelz and Pryer 2009), about one-third of all pteridophytes are epiphytic, whereas the remaining two-thirds are mostly terrestrial. Therefore, we were not surprised to find out that most pteridophytes of the RPPN are terrestrial. The numbers would be even higher if we included those terrestrial species that are either scandent (Hypolepis acantha, Lygodium volubile, and Salpichlaena volubilis) or arborescent (Cyatheaceae). Regarding the epiphytes (77 spp., including hemiepiphytes and occasional epiphytes), RPPN Guaricica is among the most species-rich areas in Brazil. For instance, Mazziero et al. (2015), Matos et al. (2010), Blum et al. (2011), and Labiak and Prado (1998) have recorded 79, 77, 74, and 69 epiphytic species, respectively. Asplenium тисronatum C.Presl, A. pteropus Kaulf., Pecluma truncorum (Lindm.) M.G.Price, Polyphlebium angustatum (Carmich.) Ebihara \& Dubuisson, and Trichomanes polypodioides L. were found exclusively on the trunks of tree ferns. This was expected, given that the preference of certain species of ferns for the trunks of Cyatheaceae has been well documented in the literature (e.g. Moran et al. 2003; Schmitt and Windisch 2005; Gasper and Sevegnani 2010; Matos et al. 2010; Mazziero et al. 2015). Most epiphytic species, however, do not depend on tree ferns and occur on a wide variety of angiosperms. For instance, Cheiroglossa palmata (L.) C.Presl, three species of Phlegmariurus Holub, and many species of Microgramma C.Presl were found exclusively on the top of angiosperm trees. These canopy epiphytes were probably poorly sampled in our study, as they were only collected when a fallen tree or tree branches were found on the ground. Eleven species were recorded as lithophytic, but most of these species were also found as either epiphytic or terrestrial. Perhaps the only obligate lithophyte is Hymenasplenium triquetrum (N.Murak. \& R.C.Moran) L.Regalado \& Prada, which is a rheophytic species. Azolla filiculoides and Salvinia auriculata Aubl. are the only floating aquatic species of ferns in the RPPN. They occur in paddy fields near the Gervásio area, in mud pools that are often used by the buffaloes, and occasionally along the Cachoeira and Faisqueira rivers.

There is a great number of pteridophyte species that are typically associated with disturbed environments such as roadsides, pastures, forest edges, forest regrowth, and other anthropogenic sites. Many of these species are non-native and invasive (e.g., Christella dentata, C. hispidula, Deparia petersenii, Macrothelypteris torresiana, Nephrolepis exaltata, Pteris ensiformis, $P$. tripartita, and P. vittata). Pteris ensiformis is native to India, China, Malaysia to Australia, and Polynesia. It has white-green variegated leaves and it is widely cultivated around the world (Hoshizaki and Moran 2001). Although it was not cited for Brazil by Prado et al. (2015), our observations have confirmed its ability to naturalize after escaping from cultivation (a healthy population was found near trilha da Pantera). This was also noted by Schwartsburd et al. (2017) in the state of Espírito Santo. The collection from RPPN Guaricica (Gomes 51, UPCB) was previously identified as Pteris multifida Poir. and appeared as such in Flora do Paraná (Kaehler et al. 2014). Another species that has escaped cultivation in the RPPN is Adiantum raddianum C.Presl, a Neotropical fern that is widely cultivated throughout the world (Mickel and Smith 2004). It was not known from RPPN Guaricica until someone brought it in a vase ca 13 years ago (J. Pontes, pers. comm.). Now it is found near many buildings and other anthropogenic sites.

Macrothelypteris torresiana, Pteris vittata (Fig. 5E), and some native Polypodiaceae (e.g. Campyloneurum nitidum (Kaulf.) C.Presl, C. rigidum J.Sm., Microgramma percussa (Cav.) de la Sota, Pleopeltis astrolepis (Liebm.) E.Fourn., and P. pleopeltifolia (Raddi) Alston) can establish themselves in the mortar-filled joints of brick walls and concrete structures, taking advantage of cracks and crannies of old buildings. Tectaria pilosa (Fée) R.C.Moran seems to have a preference for ancient 
anthropogenic sites, growing on shell middens (of unknown age) in the southern part of the RPPN (Fig. 3D, 6F). On road banks, trail sides, and other open areas, the most common species are Anemia tomentosa (Sav.) Sw. var. anthriscifolia (L.) Sw., Blechnum occidentale L., B. polypodioides Raddi, Cyclosorus interruptus (Willd.) H.Ito, Gleicheniaceae species, Lygodium volubile, Neoblechnum brasiliense (Desv.) Gasper \& V.A.O.Dittrich (Fig. 5F), Meniscium serratum Cav., Palhinhaea cernua (L.) Franco \& Vasc., Pityrogramma calomelanos (L.) Link, and Steiropteris decussata (L.) A.R.Sm.

Most species, however, have a preference for growing in the deep shade of the forests. One of the most common forest species in the RPPN is Polybotrya cylindrica (Fig. 6D). It was the dominant fern species in secondary submontane forests and, together with Danaea geniculata Raddi, represented ca $50 \%$ of all individuals found in the old-grown submontane forests of the RPPN (Gomes 2006). On the other hand, species such as Asplenium alatum Humb. \& Bonpl. ex Willd., Bolbitis serratifolia (Mert. ex Kaulf.) Schott, Elaphoglossum chrysolepis (Fée) Alston, E. paulistanum Rosenst., Hemidictyum marginatum (L.) C.Presl, Hymenophyllum microcarpum Desv., Marattia cicutifolia, Oleandra australis, Saccoloma elegans, and Selaginella valida are quite rare in the area, with only a few individuals found in remote forest fragments.

Some species are typical of certain vegetation types. Acrostichum danaeifolium Langsd. \& Fisch., for example, grows only in mangroves and other riverine environments influenced by saltwater (Figs. 3E, 5B). In the lowland sandy restinga vegetation, the most notable species are Actinostachys pennula, Cyathea atrovirens (Langsd. \& Fisch.) Domin, Lindsaea portoricensis Desv., Nephrolepis biserrata (Sw.) Schott, Serpocaulon latipes (Langsd. \& Fisch.) A.R.Sm., Telmatoblechnum serrulatum (Rich.) Perrie, D.J.Ohlsen \& Brownsey, and Trichomanes cristatum Kaulf. (Fig. 4F). Some genera, including Ctenitis (C.Chr) C.Chr., Danaea Sm., Marattia Sw., Megalastrum Holttum, Melpomene A.R.Sm. \& R.C.Moran, Oleandra Cav., Saccoloma Kaulf., and Stigmatopteris C.Chr. are typically montane. On the top of the highest mountains of the RPPN, particularly Morro do Queimado and Morro do Mirante (a.k.a. Morro do Gervásio), there are patches of cloud forest that are home to many interesting ferns, including Melpomene melanosticta (Kunze) A.R.Sm. \& R.C.Moran, Oleandra australis (Figs 3B, 6G), and Schizaea elegans (Vahl) Sw. (Fig. 4G). Also, on the very top of Morro do Mirante there is an open area that is densely covered with Dicranopteris flexuosa (Schrad.) Underw., Palhinhaea cernua, and Pteridium arachnoideum (Kaulf.) Maxon (Fig. 3A).

Despite their abundance and diversity at RPPN Guaricica, there are very few pteridophytes that can be distinguished by the local people to the point of having a vernacular name. Examples would be "avenca" (which applies to all species of Adiantum); "xaxim bugio" (howler monkey tree fern, for Alsophila sternbergii); "samambaia de renda" (lace fern, for Asplenium mucronatum); "samambaia corda-de-viola" (guitar-string fern, for Lygodium volubile); "samambaia de metro" (measuring-tape fern, for Nephrolepis spp.); "samambaia de fita" (tape fern, for Radiovittaria stipitata (Kunze) E.H.Crane); and "samambaia rabo-de-galo" (rooster-tail fern, for Trichomanes cristatum, Fig. 4F).

Our study shows that RPPN Guaricica is strikingly rich in ferns and lycophytes, highlighting the importance of this area for the conservation of these plants. Many factors might be contributing to the outstanding diversity of pteridophytes found in the present study, including the large extension of the area, its geographic location, the different vegetation types in various degrees of disturbance, and the wide range of other abiotic factors such as climate, elevation, and geomorphology within the area. We hope that our checklist provides a baseline for future research on the biology and ecology of ferns and lycophytes. With an extraordinary flora and a great infrastructure for researchers, RPPN Guaricica is one of the best places for the development of such studies in Brazil.

\section{Acknowledgements}

This study started in 2004, as an undergraduate research project conducted by the first author at Universidade Federal do Paraná (UFPR). The project was partially supported by grants from UFPR/National Treasure from Aug/2005 to Dec/2005, Fundação Araucária from $\mathrm{Jan} / 2006$ to Jul/2006, and the Brazilian National Council for Scientific and Technological Development (CNPq), from Aug/2006 to Dec/2006. It was finished under grant CNPq 155074/2018-0. We are very grateful to Marília Borgo, Reginaldo Antunes Ferreira, Ricardo Miranda de Britez, and all staff at SPVS for logistical support. Special thanks to park rangers Antônio da Veiga, João Carlos Pontes, João Maria dos Santos, Ubirajara Rodrigues Ferreira, and Vandir da Veiga for the companionship and assistance in the field. Our friends and colleagues Ana Gabriela Braga, André de Meijer, Grazielle Weiss, Jovani B. de Souza Pereira, Marcelo Reginato, Marina Caldas Gomes, Mitzi Oliveira Silva, and Pedro Bond Schwartsburd were enormously helpful to the first author during the field trips. We also thank the students of UFPR's graduate-level course on pteridology, which was taught by FM and PL at the Reserve in September 2017. While we take full responsibility for all names listed here, we are indebted to the following taxonomists for assisting us in the identification of some taxa: Claudine Mynssen (Diplazium), Jefferson Prado (Adiantum), Monica Ponce (Thelypteridaceae), and Regina Hirai (Selaginella). The authors have declared that no competing interests exist.

\section{Authors' Contributions}

FM started this study in 2004, as a scientific initiation project towards his Bachelor's Degree in Biology under the guidance of PL. FM and PL collected most of the 
specimens. AB gathered herbarium data, elaborated a database containing all collections from RPPN Guaricica, and prepared the graphs. FM identified nearly all specimens, prepared the figures, and wrote the manuscript. All authors revised the final version of the manuscript.

\section{References}

Benzing DH (1990) Vascular epiphytes. Cambridge University Press, Cambridge, 354 pp.

Benzing DH (2012) Air plants: epiphytes and aerial gardens. Cornell University Press, New York, 239 pp.

Blum CT, Roderjan CV, Galvão F (2011) Composição florística e distribuição altitudinal de epífitas vasculares da Floresta Ombrófila Densa na Serra da Prata, Morretes, Paraná, Brasil. Biota Neotropica 11 (4): 141-159. https://doi.org/10.1590/S1676-06032011 000400015

Borgo M, Silva SM (2003) Epífitos vasculares em fragmentos de Floresta Ombrófila Mista. Revista Brasileira de Botânica 26 (3): 391401. http://doi.org/10.1590/S0100-84042003000300012

Britez RM, Marques MCM (2005) Meio físico, caracterização geral. In: Marques MCM, Britez RM (Eds) História nNatural e cConservação da illha do Mel. Editora UFPR, Curitiba, 13-17.

Cervi AC, Acra LA, Rodrigues L, Train S, Ivanchechen SL, Moreira ALOR (1987) Contribuição ao conhecimento das pteridófitas de uma Mata de Araucária, Curitiba, Paraná, Brasil. Acta Biológica Paranaense 16: 77-85.

Cervi AC, Borgo M (2007) Epífitos vasculares no Parque Nacional do Iguaçu, Paraná (Brasil). Levantamento preliminar. Fontqueria 55 (51): 415-422.

Dittrich VAO, Kozera C, Silva SM (1999) Levantamento florístico dos epífitos vasculares do Parque Barigüi, Curitiba, Paraná, Brasil Iheringia Série Botânica 52: 11-21.

Dittrich VAO, Waechter JL, Salino A (2005) Species richness of pteridophytes in a montane Atlantic rain forest plot of Southern Brazil Acta Botanica Brasilica 19 (3): 519-525. http://doi.org/10.1590/ S0102-33062005000300013

Ferretti AR, Britez RM (2006) Ecological restoration, carbon sequestration, and biodiversity conservation: the experience of the Society for Wildlife Research and environmental education (SPVS) in the Atlantic Rain Forest of southern Brazil. Journal for Natural Conservation 14: 249-259. https://doi.org/10.1016/j. jnc.2006.04.006

Gasper AL, Sevegnani L (2010) Lycophyta e samambaias do Parque Nacional da Serra do Itajaí, Vale do Itajaí, SC, Brasil. Hoehnea 37 (4): 755-767. http://doi.org/10.1590/S2236-89062010000400006

Gomes MC (2006) Distribuição e diversidade de pteridófitas em diferentes estádios de regeneração florestal na Reserva Natural Rio Cachoeira, Antonina, PR. B.Sc. dissertation, Universidade Federal do Paraná, Curitiba, 59 pp.

Haufler CH, Pryer KM, Schuettpelz E, Sessa EB, Farrar DR, Moran RC, Schneller JJ, Watkins JE, Windham MD (2016) Sex and the single gametophyte: revising the homosporous vascular plant life cycle in light of contemporary research. Bioscience 66 (11): 928 937. https://doi.org/10.1093/biosci/biw108

Hirai RY, Prado J (2000). Selaginellaceae Willk. no estado de São Paulo. Brazilian Journal of Botany 23 (3): 313-339. https://doi. org/10.1590/S0100-84042000000300007

Hoshizaki BJ, Moran RC (2001) Fern grower's manual. Timber Press, Portland, $604 \mathrm{pp}$.

IBGE (2012) Manual Técnico da Vegetação Brasileira, $2^{\mathrm{a}}$ ed. Instituto Brasileiro de Geografia e Estatística, Rio de Janeiro, 271 pp.

IPARDES (1995) Diagnóstico Ambiental da APA de Guaraqueçaba. Instituto Paranaense de Desenvolvimento Econômico e Social, Curitiba, 178 pp.

IPNI (2019, continuously updated) International plant names index. http://www.ipni.org. Accessed on: 2019-10-22.

Kaehler M, Goldenberg R, Evangelista PHL, Ribas OS, Vieira AOS, Hatschbach GG (2014) Plantas vasculares do Paraná. Editora UFPR, Curitiba, 198 pp.

Kenrick P, Crane PR (1997) The origin and early diversification of land plants: a cladistic study. Smithsonian Institution Press, Washington, $441 \mathrm{pp}$.

Kottek M, Grieser J, Beck C, Rudolf B, Rubel F (2006) World map of the Köppen-Geiger climate classification updated. Meteorologische Zeitschrift 15 (3): 259-263. https://doi.org/10.1127/0941-29 48/2006/0130

Kozera C, Dittrich VAO, Silva SM (2006) Composição florística da Floresta Ombrófila Mista Montana do Parque Municipal do Barigüi, Curitiba, PR. Floresta 36 (1): 45-58. https://doi.org/10.5380/ rf.v36i1.5965

Kress WJ (1986) The systematic distribution of vascular epiphytes: an update. Selbyana 9 (1): 2-22.

Labiak PH (2014) Aspectos fitogeográficos do Paraná. In: Kaehler M, Goldenberg R, Evangelista PHL, Ribas OS, Vieira AOS, Hatschbach GG (Eds) Plantas vasculares do Paraná. Editora UFPR, Curitiba, 7-22.

Labiak PH, Prado J (1998) Pteridófitas epífitas da Reserva Volta Ve1ha, Itapoá, Santa Catarina, Brasil. Boletim do Instituto de Botânica 11: 1-79.

Labiak PH, Prado J (2008) New combinations in Serpocaulon and a provisional key for the Atlantic Rain Forest species. American Fern Journal 98 (3): 139-159. https://doi.org/10.1640/00028444(2008)98[139:ncisaa]2.0.CO;2

Lautert M, Temponi LG, Viveros RS, Salino A (2015) Lycophytes and ferns composition of Atlantic forest conservation units in western Paraná with comparisons to other areas in southern Brazil. Acta Botanica Brasilica 29 (4): 499-508. http://doi.org/10.1590/0102-3 $3062015 \mathrm{abb} 0057$

Maack R (1981) Geografia física do estado do Paraná. $2^{\mathrm{a}}$ ed. José Olympio, Rio de Janeiro, 450 pp.

Matos FB (2007) Pteridófitas da Reserva Natural Rio Cachoeira, município de Antonina, Paraná, Brasil. B.Sc. dissertation, Universidade Federal do Paraná, Curitiba, 52 pp.

Matos FB, Mickel JT (2014) The Brazilian species of Elaphoglossum section Polytrichia (Dryopteridaceae). Brittonia 66 (4): 371-395. https://doi.org/10.1007/s12228-014-9352-2

Matos FB, Amorim AM, Labiak PH (2010) The ferns and lycophytes of a montane tropical forest in southern Bahia, Brazil. Journal of Botanical Research Institute of Texas 4 (1): 333-346.

Mazziero FFF, Labiak PH, Paciencia MLB (2015) Checklist of ferns and lycophytes from the Parque Estadual Turístico do Alto Ribeira, Iporanga, São Paulo, Brazil. Check List 11 (6): 1791. https:// doi.org/10.15560/11.6.1791

Mazziero FFF, Labiak PH, Paciencia MLB (2018) Samambaias e licófitas do Parque Estadual Turístico do Alto Ribeira, Iporanga, São Paulo, Brazil. Journal of Neotropical Biology 15 (1): 22-72. https://doi.org/10.5216/rbn.v15i1.49579

Michelon C, Labiak PH (2013) Samambaias e licófitas do Parque Estadual do Guartelá, PR, Brasil. Hoehnea 40 (2): 191-204.

Michelon C, Mazziero FFF, Canestraro BK, Engels ME (2018) An illustrated guide of ferns and lycophytes from Carambeí, PR, Brazil. Rodriguésia 69 (2): 309-321. http://doi.org/10.1590/21757860201869204

Mickel JT, Smith AR (2004) The pteridophytes of Mexico. Memoirs of the New York Botanical Garden 88: 1-1054.

Moran RC (1991) Monograph of the Neotropical fern genus Stigmatopteris (Dryopteridaceae). Annals of the Missouri Botanical Garden 78 (4): 857-914. https://doi.org/10.2307/2399732

Moran RC, Riba R (1995) Psilotaceae a Salviniaceae. In: Davidse G, Souza M, Knapp S (Eds) Flora Mesoamericana, vol. 1. Universidad Nacional Autónoma de México, México, 470 pp.

Moran RC, Klimas S, Carlsen M (2003) Low-trunk epiphytic ferns on tree ferns versus angiosperms in Costa Rica. Biotropica 35 (1): 
48-56. https://www.jstor.org/stable/30043033

Moran RC, Prado J, Labiak PH (2009) Megalastrum (Dryopteridaceae) in Brazil, Paraguay, and Uruguay. American Fern Journal 99 (1): 1-44. https://doi.org/10.1640/0002-8444-99.1.1

Øllgaard B, Windisch PG (2019) Lycopodiaceae in Brazil. Conspectus of the family III. The genera Huperzia and Phlegmariurus. Rodriguésia 70: e01932017. https://doi.org/10.1590/21757860201970022

Pereira JBS, Labiak PH (2018) Checklist of ferns and lycophytes from the highlands of Pico Paraná State Park, Paraná, Brazil. Rodriguésia 69 (2): 301-307. http://doi.org/10.1590/2175-7860201869203

PPG I (Pteridophyte Phylogeny Group) (2016) A community-derived classification for extant lycophytes and ferns. Journal of Systematics and Evolution 54 (6): 563-603. https://doi.org/10.1111/ jse. 12229

Prado J, Windisch PG (2000) The genus Pteris L. (Pteridaceae) in Brazil. Boletim do Instituto de Botânica 13: 103-199.

Prado J, Sylvestre LS, Labiak PH, Windisch PG, Salino A, Barros ICL, Hirai RY, Almeida TE, Santiago ACP, Kieling-Rubio MA, Pereira AFN, Øllgaard B, Ramos CGV, Mickel JT, Dittrich VAO, Mynssen CM, Schwartsburd PB, Condack JPS, Pereira JBS, Matos FB (2015) Diversity of ferns and lycophytes in Brazil. Rodriguésia 66 (4): 1073-1083. https://doi.org/10.1590/21757860201566410

Pryer KM, Schneider H, Smith AR, Cranfill R, Wolf PG, Hunt JS (2001) Horsetails and ferns are a monophyletic group and the closest living relatives to seed plants. Nature 409: 618-621. https://doi.org/10.1038/35054555

Reflora (2019) http://reflora.jbrj.gov.br/reflora/herbarioVirtual/. Accessed on: 2019-10-22.

Salino A, Almeida TE (2008) Pteridófitas do Parque Estadual do Jacupiranga, SP, Brasil. Acta Botanica Brasilica 22 (4): 983-991. https://oi.org/10.1590/S0102-33062008000400009

Salino A, Silva SM, Dittrich VAO, Britez RM (2005) Flora pteridofítica. In: Marques MCM, Britez RM (Eds) História Natural e Conservação da Ilha do Mel. Editora UFPR, Curitiba, 85-101.
Schmitt JL, Windisch PG (2005) Aspectos ecológicos de Alsophila setosa Kaulf. (Cyatheaceae, Pteridophyta) no Rio Grande do Sul, Brasil. Acta Botanica Brasilica 19 (4): 859-865. https://doi.org/10. 1590/S0102-33062005000400021

Schuettpelz E, Pryer K (2009) Evidence for a Cenozoic radiation of ferns in an angiosperm-dominated canopy. Proceedings of the National Academy of Sciences 106 (27): 11200-11205. https://doi. org/10.1073/pnas.0811136106

Schwartsburd PB (2012) Three new taxa of Hypolepis (Dennstaedtiaceae) from the Brazilian Atlantic Forest, and a key to the Brazilian taxa. Kew Bulletin 67 (4): 815-825. https://doi.org/10.1007/s 12225-012-9402-4

Schwartsburd PB, Labiak PH (2007) Pteridófitas do Parque Estadual de Vila Velha, Ponta Grossa, Paraná, Brasil. Hoehnea 34 (2): 159 209. http://doi.org/10.1590/S2236-89062007000200005

Schwartsburd PB, Miranda CV, Prado J (2016) Oleandra (Oleandraceae) in the Brazilian Atlantic forest. American Fern Journal 106 (3): 191-205. https://doi.org/10.1640/AFJ-D-16-00004.1

Schwartsburd PB, Miranda CV, Pena NTL, Oliveira MH, Silva RV, Marcolino F (2017) Checklist of ferns and lycophytes from Parque Estadual Mata das Flores, Espírito Santo, Brazil. Check List 13 (5): 621-633. https://doi.org/10.15560/13.5.621

Souza FS, Salino A, Viana PL, Salimena FRG (2012) Pteridófitas da Serra Negra, Minas Gerais, Brasil. Acta Botanica Brasilica 26 (2): 378-390. http://doi.org/10.1590/S0102-33062012000200013

SpeciesLink (2019) http://www.splink.org.br/index. Accessed on: 201910-22.

Thiers B (2019, continuously updated) Index Herbariorum: a worldwide index of herbaria and associated staff. http://sweetgum. nybg.org/science/ih/. Accessed on: 2019-10-22.

Veloso HP, Rangel-Filho ALR, Lima JCA (1991) Classificação da vegetação brasileira adaptada a um sistema universal. IBGE, Rio de Janeiro, 123 pp.

Viveros RS, Rouhan G, Salino A (2018) A taxonomic monograph of the fern genus Ctenitis (Dryopteridaceae) in South America. Phytotaxa 385 (1): 1-83. https://doi.org/10.11646/phytotaxa.385.1.1

\section{Appendix}

Table A1. Sampling sites and their corresponding vegetation types in Reserva Natural Guaricica.

\begin{tabular}{|c|c|c|}
\hline Sampling site & Coordinates & Vegetation types \\
\hline 1. Estrada Cacatu - Guaraqueçaba (PR-405) & $25^{\circ} 18^{\prime} 48^{\prime \prime} \mathrm{S}, 048^{\circ} 41^{\prime} 38^{\prime \prime} \mathrm{W}$ & Anthropogenic habitats; Pasture / Open areas; Secondary submontane forests. \\
\hline 2. Estrada Sede - Gervásio & $25^{\circ} 17^{\prime} 33^{\prime \prime} \mathrm{S}, 048^{\circ} 42^{\prime} 15^{\prime \prime} \mathrm{W}$ & Anthropogenic habitats; Pasture / Open areas; Secondary submontane forests. \\
\hline 3. Faisqueira & $25^{\circ} 22^{\prime} 14^{\prime \prime} \mathrm{S}, 048^{\circ} 39^{\prime} 56^{\prime \prime} \mathrm{W}$ & Riparian vegetation; Mangroves; Secondary submontane forests. \\
\hline 4. Fazenda Cachoeira & $25^{\circ} 18^{\prime} 53^{\prime \prime} \mathrm{S}, 048^{\circ} 41^{\prime} 46^{\prime \prime} \mathrm{W}$ & Anthropogenic habitats; Pasture / Open areas; Riparian vegetation; Secondary submontane forests \\
\hline 5. Fazenda Rincão (Sede administrativa) & $25^{\circ} 18^{\prime} 53^{\prime \prime} \mathrm{S}, 048^{\circ} 41^{\prime} 46^{\prime \prime} \mathrm{W}$ & Anthropogenic habitats; Pasture / Open areas; Riparian vegetation; Secondary submontane forests \\
\hline 6. Fazenda Santa Olímpia & $25^{\circ} 16^{\prime} 45^{\prime \prime} \mathrm{S}, 048^{\circ} 43^{\prime} 57^{\prime \prime} \mathrm{W}$ & Anthropogenic habitats; Pasture / Open areas; Secondary submontane forests. \\
\hline 7. Margem esquerda do Rio Cachoeira & $25^{\circ} 23^{\prime} 05^{\prime \prime} \mathrm{S}, 048^{\circ} 43^{\prime} 04^{\prime \prime} \mathrm{W}$ & Pasture / Open areas; Riparian vegetation (arboreal and herbaceous). \\
\hline 8. Margem esquerda do Rio Cupiúva & $25^{\circ} 18^{\prime} 46^{\prime \prime} \mathrm{S}, 048^{\circ} 40^{\prime} 16^{\prime \prime} \mathrm{W}$ & Pasture / Open areas; Secondary submontane forests. \\
\hline 9. Morro do Queimado & $25^{\circ} 23^{\prime} 10^{\prime \prime} \mathrm{S}, 048^{\circ} 42^{\prime} 19^{\prime \prime} \mathrm{W}$ & Old-grown montane forest. \\
\hline 10. Porto do Cachaça & $25^{\circ} 20^{\prime} 27^{\prime \prime S}, 048^{\circ} 41^{\prime} 47^{\prime \prime} \mathrm{W}$ & Riparian vegetation (arboreal). \\
\hline 11. Trilha dos 600 metros & $25^{\circ} 14^{\prime} 23^{\prime \prime} \mathrm{S}, 048^{\circ} 39^{\prime} 24^{\prime \prime} \mathrm{W}$ & Old-grown submontane forests. \\
\hline 12. Trilha das Arapongas & $25^{\circ} 18^{\prime} 11^{\prime \prime S}, 048^{\circ} 41^{\prime} 10^{\prime \prime} \mathrm{W}$ & Pasture / Open areas; Secondary submontane forests. \\
\hline 13. Trilha da Baixada & $25^{\circ} 14^{\prime} 54^{\prime \prime} \mathrm{S}, 048^{\circ} 40^{\prime} 37^{\prime \prime} \mathrm{W}$ & Secondary submontane forests. \\
\hline 14. Trilha do Bezerra & $25^{\circ} 19^{\prime} 25^{\prime \prime} \mathrm{S}, 048^{\circ} 41^{\prime} 26^{\prime \prime} \mathrm{W}$ & Pasture / Open areas; Riparian vegetation (arboreal and herbaceous). \\
\hline 15. Trilha Caixa d'Água & $25^{\circ} 23^{\prime} 19^{\prime \prime} \mathrm{S}, 048^{\circ} 40^{\prime} 05^{\prime \prime} \mathrm{W}$ & Mangroves; Secondary submontane forests. \\
\hline 16. Trilha do Calé & $25^{\circ} 20^{\prime} 26^{\prime \prime} \mathrm{S}, 048^{\circ} 40^{\prime} 50^{\prime \prime} \mathrm{W}$ & Secondary submontane forests. \\
\hline 17. Trilha do Corvo & $25^{\circ} 19^{\prime} 39^{\prime \prime} \mathrm{S}, 048^{\circ} 40^{\prime} 26^{\prime \prime} \mathrm{W}$ & Secondary submontane forests. \\
\hline 18. Trilha Esborracha Faisqueira & $25^{\circ} 23^{\prime} 37^{\prime \prime} \mathrm{S}, 048^{\circ} 40^{\prime} 52^{\prime \prime} \mathrm{W}$ & Secondary submontane forests. \\
\hline 19. Trilha da Estrada & $25^{\circ} 17^{\prime} 17^{\prime \prime} \mathrm{S}, 048^{\circ} 38^{\prime} 40^{\prime \prime} \mathrm{W}$ & Secondary submontane forests. \\
\hline 20. Trilha dos Fornos & $25^{\circ} 17^{\prime} 38^{\prime \prime} \mathrm{S}, 048^{\circ} 39^{\prime} 25^{\prime \prime} \mathrm{W}$ & Pasture / Open areas; Secondary submontane forests. \\
\hline 21. Trilha do Gervásio & $25^{\circ} 14^{\prime} 39^{\prime \prime} \mathrm{S}, 048^{\circ} 39^{\prime} 59^{\prime \prime} \mathrm{W}$ & Pasture / Open areas; Secondary submontane forests; Old-grown submontane forests. \\
\hline 22. Trilha da Guaricica & $25^{\circ} 19^{\prime} 13^{\prime \prime} \mathrm{S}, 048^{\circ} 42^{\prime} 04^{\prime \prime} \mathrm{W}$ & Pasture / Open areas; Secondary submontane forests. \\
\hline 23. Trilha do Jonata & $25^{\circ} 21^{\prime} 40^{\prime \prime} \mathrm{S}, 048^{\circ} 41^{\prime} 34^{\prime \prime} \mathrm{W}$ & Riparian vegetation (arboreal); Secondary submontane forests. \\
\hline 24. Trilha do Macaco & $25^{\circ} 15^{\prime} 00^{\prime \prime} \mathrm{S}, 048^{\circ} 39^{\prime} 25^{\prime \prime} \mathrm{W}$ & Secondary submontane forests; 0ld-grown submontane forests. \\
\hline 25. Trilha do Matozo & $25^{\circ} 20^{\prime} 38^{\prime \prime} \mathrm{S}, 048^{\circ} 41^{\prime} 12^{\prime \prime} \mathrm{W}$ & Riparian vegetation (arboreal); Secondary submontane forests. \\
\hline
\end{tabular}


Table A1. Continued.

\begin{tabular}{|c|c|c|}
\hline Sampling site & Coordinates & Vegetation types \\
\hline 26. Trilha do Meio & $25^{\circ} 14^{\prime} 38^{\prime \prime} \mathrm{S}, 048^{\circ} 39^{\prime} 13^{\prime \prime} \mathrm{W}$ & Pasture / Open areas; Secondary submontane forests; Old-grown submontane forests. \\
\hline 27. Trilha do Mergulhão & $25^{\circ} 17^{\prime} 36^{\prime \prime} \mathrm{S}, 048^{\circ} 43^{\prime} 57^{\prime \prime} \mathrm{W}$ & Anthropogenic habitats; Pasture / Open areas; Secondary submontane forests. \\
\hline 28. Trilha do Mirante & $25^{\circ} 14^{\prime} 25^{\prime \prime} \mathrm{S}, 048^{\circ} 39^{\prime} 55^{\prime \prime} \mathrm{W}$ & Secondary submontane forests; 0ld-grown submontane and montane forests. \\
\hline 29. Trilha do Neno & $25^{\circ} 22^{\prime} 36^{\prime \prime} \mathrm{S}, 048^{\circ} 40^{\prime} 10^{\prime \prime} \mathrm{W}$ & Secondary submontane forests. \\
\hline 30. Trilha da Pantera & $25^{\circ} 18^{\prime} 02^{\prime \prime} \mathrm{S}, 048^{\circ} 38^{\prime} 31^{\prime \prime} \mathrm{W}$ & Secondary submontane forests; Old-grown submontane forests. \\
\hline 31. Trilha dos Pinheiros & $25^{\circ} 20^{\prime} 05^{\prime \prime} \mathrm{S}, 048^{\circ} 40^{\prime} 54^{\prime \prime} \mathrm{W}$ & Secondary submontane forests; Old-grown submontane forests. \\
\hline 32. Trilha Porto Faisqueira & $25^{\circ} 21^{\prime} 58^{\prime \prime} \mathrm{S}, 048^{\circ} 39^{\prime} 25^{\prime \prime} \mathrm{W}$ & Pasture / Open areas; Secondary submontane forests. \\
\hline 33. Trilha Porto do Rio Seco & $25^{\circ} 24^{\prime} 19^{\prime \prime} \mathrm{S}, 048^{\circ} 40^{\prime} 54^{\prime \prime} \mathrm{W}$ & Mangroves; Secondary submontane forests \\
\hline 34. Trilha do Queimado & $25^{\circ} 23^{\prime} 03^{\prime \prime} \mathrm{S}, 048^{\circ} 42^{\prime} 06^{\prime \prime} \mathrm{W}$ & Secondary lowland forests; Secondary submontane forests. \\
\hline 35. Trilha da Rede & $25^{\circ} 17^{\prime} 49^{\prime \prime} \mathrm{S}, 048^{\circ} 40^{\prime} 23^{\prime \prime} \mathrm{W}$ & Pasture / Open areas; Secondary submontane forests. \\
\hline 36. Trilha Rio do Ferro & $25^{\circ} 18^{\prime} 07^{\prime \prime} \mathrm{S}, 048^{\circ} 40^{\prime} 49^{\prime \prime} \mathrm{W}$ & Pasture / 0 pen areas; Secondary submontane forests; Old-grown submontane forests. \\
\hline 37. Trilha Rio do Turvo & $25^{\circ} 17^{\prime} 14^{\prime \prime} \mathrm{S}, 048^{\circ} 39^{\prime} 54^{\prime \prime} \mathrm{W}$ & Pasture / Open areas; Secondary submontane forests; Old-grown submontane forests. \\
\hline 38. Trilha do Taquaral & $25^{\circ} 22^{\prime} 44^{\prime \prime} \mathrm{S}, 048^{\circ} 41^{\prime} 58^{\prime \prime} \mathrm{W}$ & Riparian vegetation; Secondary lowland forests; Secondary submontane forests. \\
\hline 39. Trilha do Zé Carlos & $25^{\circ} 19^{\prime} 49^{\prime \prime} \mathrm{S}, 048^{\circ} 39^{\prime} 14^{\prime \prime} \mathrm{W}$ & Secondary submontane forests; Old-grown submontane forests. \\
\hline
\end{tabular}

